\title{
Design and Implementation of an Electronic System of Salaries: (Nineveh Investment Commission as a Model)
}

\author{
Mohamed Q. Al-Chalabi ${ }^{1 *}$, Mafaz M. Al-Anezi² \\ ${ }^{1 *}$ Nineveh Investment Commission, Mosul, Iraq \\ ${ }^{2}$ Department of Computer Science, College of Computer Science and Mathematics, University of Mosul, \\ Mosul, Iraq \\ E-mail: ${ }^{1 *}$ alchalabimohamed@gmail.com, ${ }^{2}$ mafazmhalanezi@ uomosul.edu.iq
}

(Received February 27, 2021; Accepted May 10, 2021; Available online September 01, 2021)

DOI: 10.33899/edusj.2021.129618.1146, (C) 2021, College of Education for Pure Science, University of Mosul.

This is an open access article under the CC BY 4.0 license (http://creativecommons.org/licenses/by/4.0/).

\begin{abstract}
:
Electronic systems are considered one of the most important pillars in the development of the work of any institution, especially the systems related to the administrative and financial aspects.

In this research, an electronic system for salaries for the Nineveh Investment Commission (NIC) was designed and implemented model using the language (C\#), A central database was built using a Database Management System (SQL), This system was based on a local wireless network to share work by adopting (Client/Server) model to connect the computers, the proposed system includes very important features such as the open system data that enables the user to add and amend the percentages of the basic and secondary salary components, automatic calculation of the salary by specifying the employee service specifications and the certificate obtained, fixed and variable allocations and deductions, calculating all leave, Determining annual bonuses and promotions and organizing them to makes it easy for the user to know who is eligible, update and calculate them, in this system several levels of system users were built. A report was added for the employee's last salary certificate with detailed reports on salaries and the system was strengthened with the feature of backing up to prevent the database from Damage and referred to at any time.

The system was tested on real data to issuing salary reports for three months. As the system met with great desire and reliability in its use by conducting a questionnaire to measure the usability of the system on the specialists.
\end{abstract}

Keywords: Databases, SQL SERVER 2008, Electronic Accounting, Payroll, LAN.

$$
\begin{aligned}
& \text { تصميم وتنفيذ نظام إلكتروني للرواتب: (هيئة استثمار نينوى أنموذجاً) } \\
& \text { محمد قصي عبدالقادر الجلبي"1، أ.م.د مفاز محسن خليل العنزي² }
\end{aligned}
$$

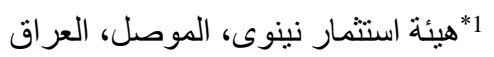

$$
\begin{aligned}
& \text { 2قنم علوم الحاسوب، كلية علوم الحاسوب و الرياضيات، جامعة الموصل، الموصل، العراق }
\end{aligned}
$$


تعد النظم الإكترونية من أهم الدعائم في تطوير عمل أي مؤسسة لاسيما المتعلقة بالجوانب الإدارية والمالية. تم في هذا البحث تصميم وتتفيذ نظام إلكتروني للرواتب لهيئة استثمار نينوى (CIC) باستخدام لغة (C\#)، تم بناء قاعدة بيانات

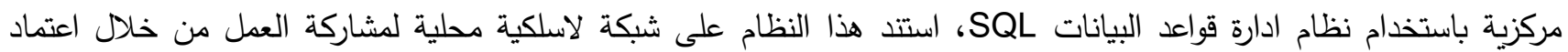
نموذج Client/Server لتوصيل أجزة الكمبيوتر ، ويتضمن النظام المقترح ميزات مهمة جدًا مثل بيانات النظام المفتوح التي تمكن بتهن

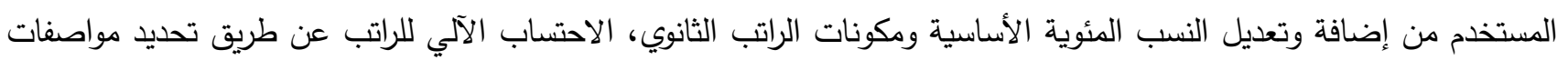

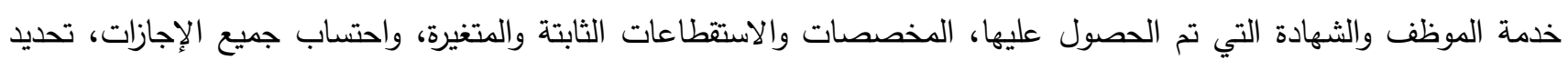
العلاوات السنوية والترفيعات وتتظيمها بطريقة تسهل على المستخدم معرفة المستحقين وتحديثها وإحتسابها، كما تم في هذا النظام بناء عدة مستويات لمستخدمي النظام. إضافة تقرير خاص بشهادة آخر راتب للموظف مع تقارير تفصيلية بالرواتب لجميع الموظفين

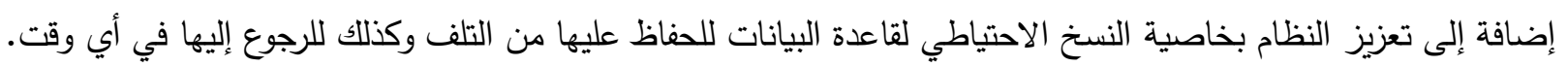
تم اختبار النظام على بيانات حقيقية لإصدار تقارير الرواتب لمدة ثلاثة أشهر • إذ لقي النظام رغبة وموثوقية كبيرة في استخدامه عن طريق عمل استبيان لمقياس قابلية استخدام النظام على الموظفين المختصين.

\section{الكلمات المفتاحية: قواعد الببانات، SQL SERVER 2008 المحاسبة الإلكترونية، الرواتب، شبكة محلية.}

1

نظراً للتطور الحاصل الذي يشهده العالم اليوم والحاجة الماسة إلى استخدام تكنلوجيا الحاسبات في جميع مجالات الحياة ومنها مؤسساتتا الحكومية، ففي بداية المحاسبة الإكترونية كان برنامج الـ Excel التطبيق الأساسي الوحيد الذي يتم من خلالده فقط أداء

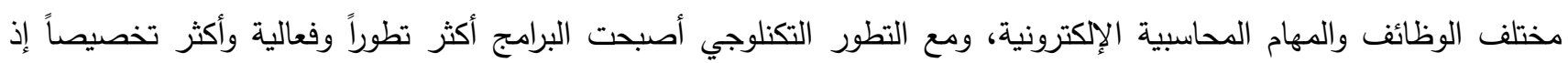
تدعم الشركات حسب حجم الشركة [3]. مع ظهور وتطور الأجهزة الإلكترونية وفي مقدمتها الحاسوب أدخلت أساليب جديدة وحديثة إلى المؤسسات الحكومية لتواكب

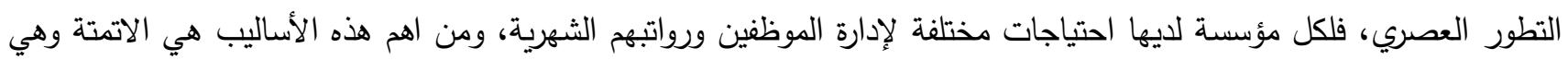

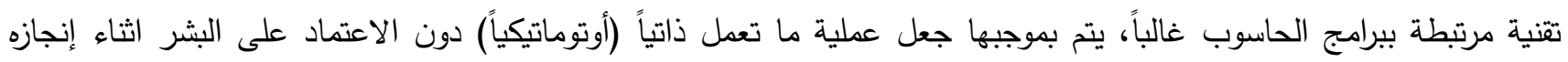

ان إدارة الرواتب عملية تستغرق وقتاً طويلاً وتتكرر بوتيرة دورية، لذا لتجنب العمل المضني والاخطاء البشرية، من المككن

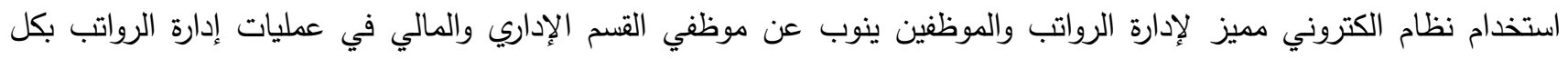

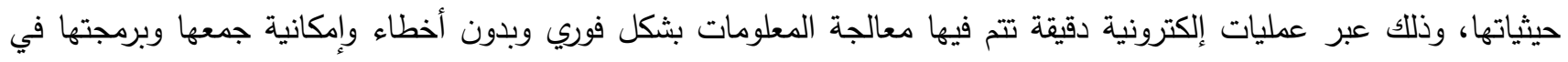

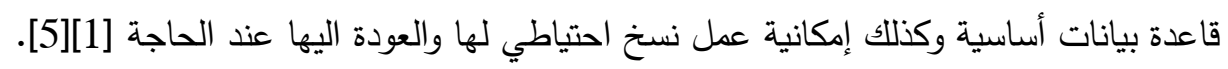

2 2. 2 أهمية البحث

يعد هذا البحث من البحوث الرائدة في مجال المحاسبة الإلكترونية في العراق، إذ تتبع أهمية البحث في تصميم نظام الكتروني للرواتب وتطبيقه على هيئة استثمار نينوى لتعزيز مشروع الحكومة الإكترونية في جميع مؤسسات الدولة العراقية وإمكانية تطبيقه لئه على هذه المؤسسات، إذ سيؤدي إلى تطوير النظام الالكتروني للرواتب لديها، والذي بدوره يقلل التكاليف ورفع مستوى الأداء والسرعة لتئه

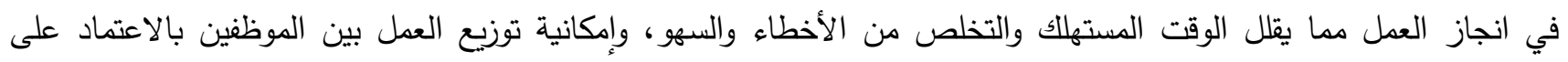
الثبكة المحلية التي تربط حاسبات المخولين، إضافة إلى ذلك إمكانية عمل نسخ احتياطي لبيانات النظام إذ يعطي أهمية كبيرة في إمكانية استعادة البيانات في حال تعرضت حاسبة الخادم للتلف والرجوع إلى بيانات الأشهر السابقة عند الحاجة اليها، كما تعد هذه الدراسة حافزاً للبدء في عمليات التطوير التقني في جميع المجالات الإدارية. 
يهذف هذا البحث إلى تصميم وبناء نظام حاسوبي متكامل لحساب رواتب الموظفين في هيئة استثمار نينوى، مستنداً على شبكة محلية في تتظيم الرواتب واستحقاقات الموظفين في القسم الإداري والمالي في الهيئة، وسرعة الرجوع إليها، وتتلخص أبرز أهدافه بالمحاور الآتية: أ. لتصميم نظام إلكتروني رصين قادر على حساب رواتب الموظفين وجميع استحقاقاتهم الإدارية والمالية إلكترونياً وبنظام مفتوح اذ

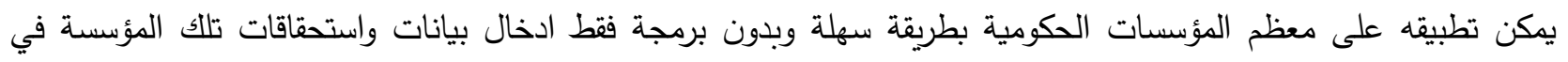
بيانات النظام، اضافةً الى احتساب العلاوات والترفيعات والاجازات بسهولة ودقة. ب. لبناء قاعدة بيانات مركزية تحتوي على معلومات الموظفين ورواتبهم وخدمتهم الوظيفية في القسم الإداري والمالي للهيئة يسهل

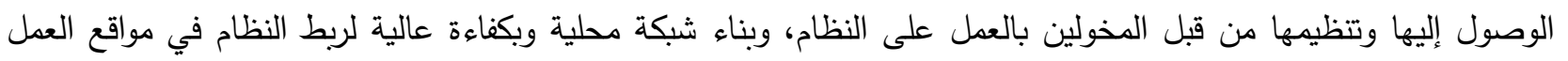

$$
\text { المختلفة حسب إحتياجات الهيئة. }
$$

ت. لعمل نسخ إحتياطي لقاعدة البيانات لحمايتها من التلف أو الفقد والرجوع إليها عند تضرر النظام أو الحاسبة المستخدمة وعند الحاجة لبيانات للأشهر السابقة. 4. مشكلة البحث جاءت مشكلة البحث من الواقع التقليدي الذي تعمل عليه معظم المؤسسات الحكومية في العراق، وهيئة استثمار نينوى غير

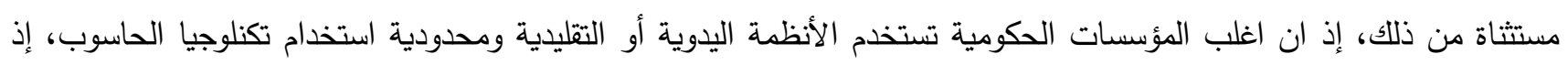
أصبحت أنظمة قديمة ولا يمكن الاعتماد عليها مع زيادة عدد الموظفين في الدوائر الحكومية الكبيرة والمتوسطة بالمقارنة مع النظم الإنة

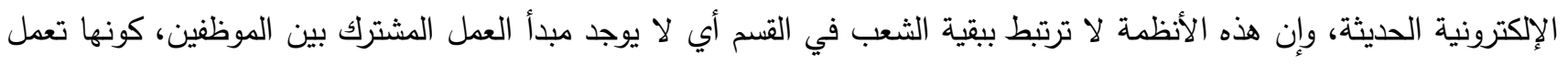

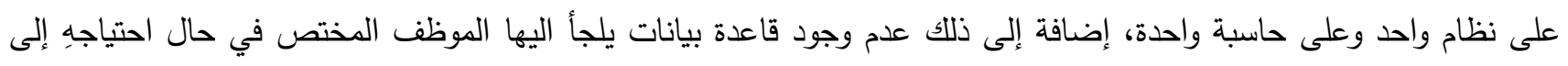
بيانات للأشهر السابقة. 5. الاراسات ذات الصلة ه 1.5 هي عام (2009) تم إعداد دراسة من قبل الباحث منذر يحيى الداية بعنوان "أثر استخدام نظم المعلومات المحاسبية على جودة

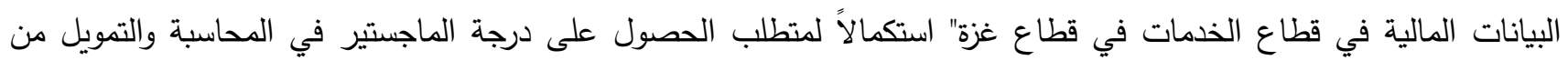
الجامعة الإسلامية في غزة كلية التجارة قسم المحاسبة والتمويل. تهدف الدراسة إلى تقييم أثر استخدام نظم المعلومات المحاسبية

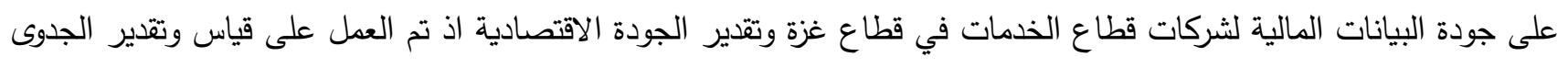
الاقتصادية من استخدام نظم المعلومات المحاسبية [26]. ه قدمت هذه الرسالة في عام (2010) من قبل الباحث حمد فهذ دعسان المطيري بعنوان "تطبيق نظم محاسبة الموارد البشرية في

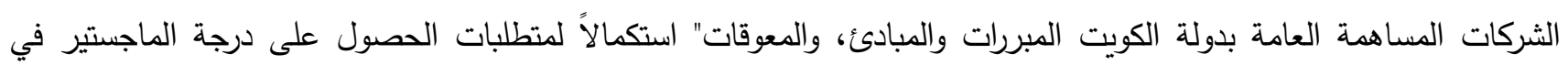
المحاسبة في الكويت جامعة الثرق الأوسط كلية الأعمال قسم المحاسبة. تهدف هذه الدراسة على التعرف على أهمية نظم المحاسبة عن الموارد البشرية ومبررات تطبيقها في الثركات المساهمة في الكويت وإلى التعرف على المبادئ والقواعد التي تحكم نظم المحاسبة على الموارد البشرية في حالة تطبيقها واستكثاف المعوقات التي تحول دون تطبيق نظم المحاسبة على الموارد البشرية في

$$
\text { الشركات المساهمة بدولة الكويت [24]. }
$$

في العام نفسه نشر الأستاذ جان سيريل فضل الله في مجلة التقنيات، المجلد 23، العدد 6، نظام بعنوان "تصميم وإعداد نظام لإدارة قاعدة البيانات الخاصة بالعاملين في جامعة بغداد". نشر تصميم نظام يمكن من خلاله توضيح وتعريف هياكل ملفات قاعدة 
البيانات، ومن ثم تعريف الحقول وتحديد أحجامها وأنواعها وإمكانية استعمالها كمفاتيح، كما يعمل النظام على توفير إمكانية تحديث

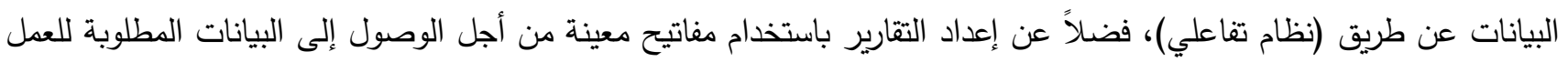

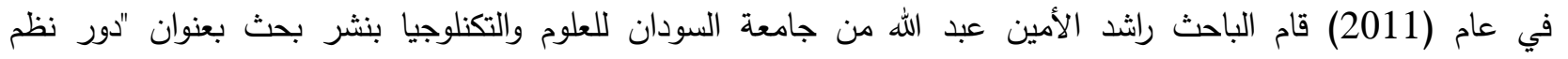
المعلومات المحاسبية المحوسبة في رفع كفاءة الأداء المالي: دراسة حالة وزارة الداخلية السودانية" كجزء من متطلبات نيل شهادة الماجستير • يهدف البحث إلى معرفة دور نظم المعلومات المحاسبية المحوسبة ورفع كفاءة الأداء المالي لوزارة الداخلية ومواكبة

$$
\text { التطور التقني باستخدام الحاسوب [23]. }
$$

هاءت رسالة الباحث وليد عبد الله عبد العزيز في عام (2017) بعنوان "أثر تطبيق نظام إدارة المعلومات المالية الحكومية على المى كفاءة إدارة المالية العامة" في مصر لاستكمال متطلبات الحصول على درجة الماجستير من معهد التخطيط القومي في مصر. تهدف الدراسة لبناء نظام إلكتروني يربط كافة أجزة الحكومة يقوم بأداء العمليات المالية المحاسبية التي تتم بكل مراحل داهل دورة

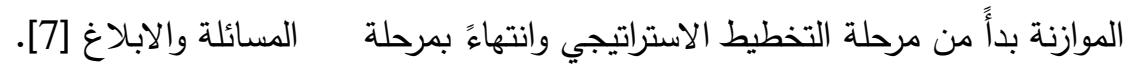
قام الباحث خليل أحمد نهير محل رسالة دبلوم عالٍ في جامعة الموصل كلية الإدارة والاقتصاد قسم إدارة الأعمال رسالته الموسومة "تصميم برنامج إلكتروني لاحتساب العلاوات والترفيعات نموذج مقترح في بعض كليات جامعة الموصل" في سنة (2019). تهدف الدراسة إلى تقديم إطار مفاهيمي لنظام التعويضات ومكوناته والآليات المستخدمة لاحتساب العلاوات والترفيعات

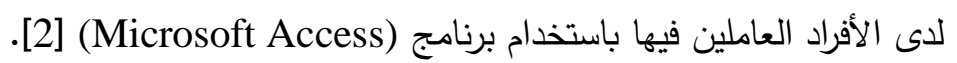

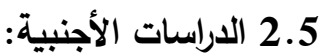

قام الباحث Ratul Paul من جامعة The people's university of Pangladesh بتصميم وتطوير نظام عن إدارة الرواتب قائم على الويب لعام (2012)، يقوم باحتساب رواتب الموظفين والمكافئات والقروض مع الاستقطاعات باستخدام لغة لنة

مع MYSQ] هramyaj Zutam من جامعة دلهي الهندية الذي قام بدراسة عن نظام خاص بإدارة الموارد البشرية يقوم بحساب الرواتب أبرات واجازات الموظفين إلكترونياً عام (2012). يهدف المشروع إلى تطوير نظام لإدارة الإجازات عبر الإنترنت يكون ذا أهمية لأي

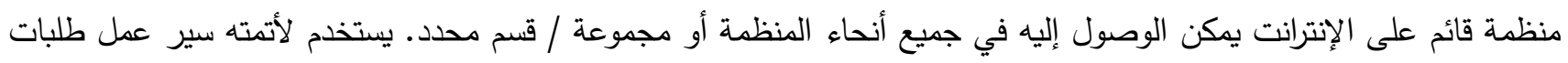

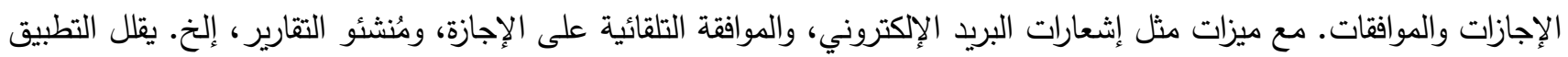
إدارة الإجازات من العمل الورقي ويحافظ على السجل بطريقة أكثر كفاءة [18]. • وفي عام (2015) قام Pronoti RoI من جامعة Lingaya's University الهندية بتصميم نظام مراجعة الرواتب المحوسب. يهدف النظام إلى إدارة كشوفات الراتب بتتبع ساعات العمل والاحتفاظ بسجل لبيانات الموظفين المتضمنة رواتب والاستقاعات والضرائب على أساس شهري ويمكن كل موظف في إدارة الموارد البشرية للدخول واجراء التعديلات على البيانات. تم

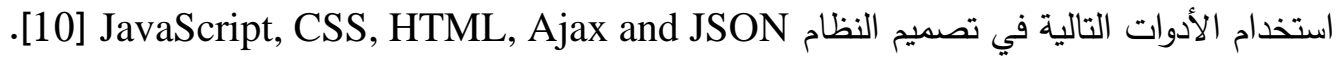
في عام (2016) قام مجموعة من الطلاب في جامعة Lagos State University, Nigeria بدراسة بعنوان Structural Determinants of Job Satisfaction: The Mutual Influences of Compensation Management and الأبعاد الهيكلية للرضا الوظيفي: التأثيرات المتبادلة لإدارة التعويضات وتحفيز الموظفين. سعت الدراسة إلى فهم آلية تحقق الرضا الوظيفي في إدارة أعمال التأمين من خلال التحقيق في الأبعاد الهيكلية لإنجاز المهمة كما هدفت الدراسة إلى تتييم التأثيرات المتبادلة لإدارة التعويضات ودوافع الموظفين للوصول إلى الرضا لـإلى الوظيفي من خلال استخدام التحليل

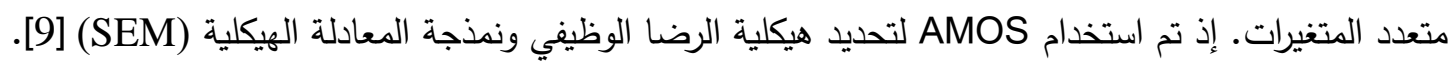


• في عام (2017) قدم الباحث Kolawole Emmanuel من جامعة Caleb University في ولاية Lagos بناً DESIGN AND IMPLEMENTATION OF EMPLOYEEINFORMATION AND PAYROLL "بعنوان SASTEM يهدف إلى بناء نظام يقوم بحساب رواتب الموظفين وبناء قاعدة بيانات للمؤسسة واستخدم جامعة كالب كبيئة عمل ANA مستخدماً لغة JAVA لتصميم الواجهات و MySQL لبناء قاعدة البيانات [8]. • وفي عام (2018) قام MARCUS ATISH D ROZARIO من معهد RCC لتكنولوجيا المعلومات التابع لجامعة مولانا أبو الآزاد للتكنولوجيا بتصميم تطبيق بشأن قاعدة بيانات الموظفين ونظام إدارة سجل الرواتب. الغرض منه إدارة شؤون الموظفين وكشوف رواتبهر عبر تطبيق ويب الأدوات والمستخدمة كانت:RDBMS: و Languages: HTML, PHP, JavaScript

.[6] Development Platform: Adobe Dreamweaver. و Web Server: WAMP server. و Online MySQL. إن الفرق بين هذه الدراسة والدراسات السابقة بأنها تناولت تصميم نظام إلكتروني للرواتب، يعمل على حساب رواتب الموظفين

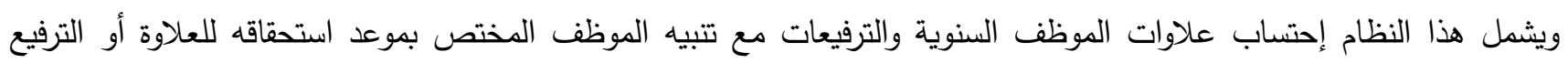

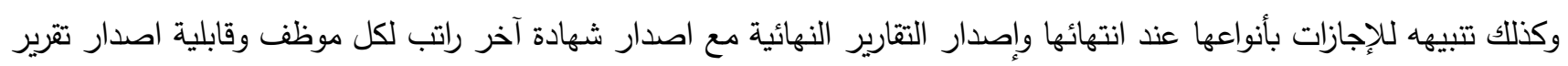
مفصل عن مفردات راتب الموظف وكذلك إمكانية النظام على إحتساب كتب الثكر والعقوبات التي يمكن للموظف الحصول عليها، فضلاً عن إن بيئة النظام تختلف عن بيئات الأنظمة المتتاولة في الدراسات البكات السابقة. 6. مفهوم نظام الرواتب

مفهوم نظام الرواتب بصورة عامة هي حساب رواتب الموظفين وجميع استحقاقاتهم الإدارية والمالية على أساس معادلات معينة والتي تتضمن إضافات واستقطاعات الموظف في كل شهر وجميع المتغيرات الأخرى من ترفيع وعلاوة التي يستحصل عليها الموظف في ذلك الشهر وغيرها من المتغيرات على الراتب وإصدار قوائم نهائية برواتب الموظفين وعملية ارشفتها [22].

7. مقارنة بين النظام المحاسبي اليدوي والنظام المحوسب 1.7 النظام المحاسبي اليدوي: وهي الأنظمة التي لا تستخدم أجهزة الحاسوب في إجراء العمليات المُحاسبية، إنما يتم تسجيلها يدوياً في دفتر اليومية وتحفظ هذه المستندات كأرشيف ورقي لكل شهر منصرم في المؤسسة، ويقوم المحاسب بإجراء جميع الحسابات الئاء

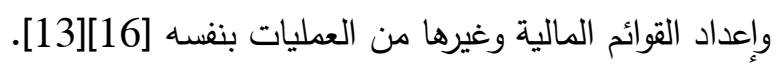

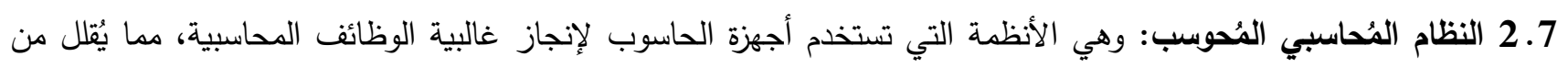

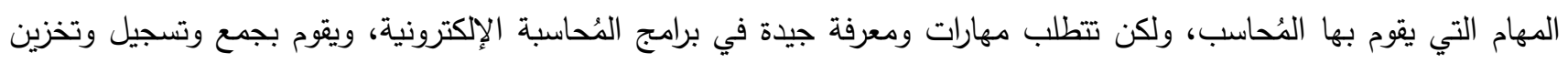

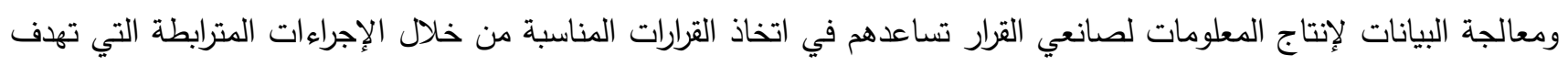
سوياً إلى تحقيق غايات محددة [13][12]. 8. ت نظام إدارة قواعد البيانات هو عبارة عن برنامج لإدارة قواعد البيانات وإنشائها والتعديل عليها، أي هو أداة المستخدم في فعل ما يشاء في أي بيانات على ألى حسب إمكانيات البرنامج الهدف منها هو توفير البيئة المناسبة والفعالة لتجميع هذه المعلومات وفئات وفرزها وتبويبها وتخزينها وحذفها واستخدامها واسترجاعها [4]. والثكل (1)، يوضح هيكلية نظام إدارة قواعد البيانات Data Base Management System: 


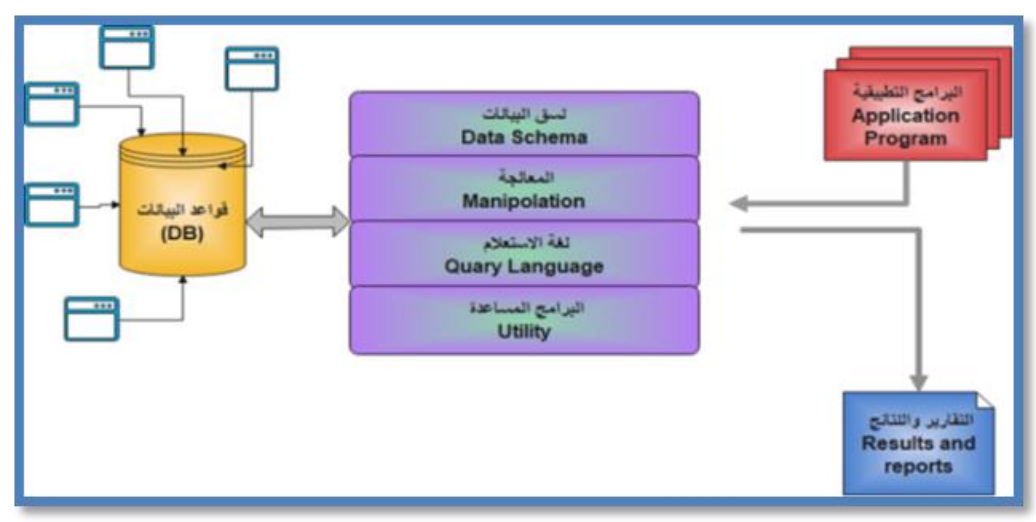

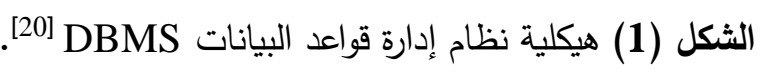

Microsoft SQL Server 2008 نظام 9.

وهو خادم قاعدة البيانات العلائقية أنتج من قبل شركة Microsoft يتم استخدامه لتخزين واسترجاع المعلومات من التطبيقات

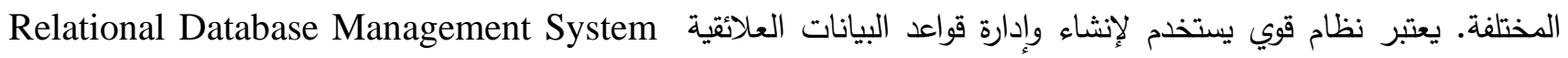
(RDBMS) ، ويحتوي هذا النظام على مجموعة كبيرة من المميزات والتي تجعله من أقوى الأنظمة المستخدمة لإنشاء وإدارة قواعد البيانات [11][11]].

\section{Backup 10. النسخ الاحتياطي}

في حالة حدوث كارثة في المكان الذي تتواجد فيه قاعدة البيانات أو حدث أي ضرر بالخادم تتيح هذه العملية استعادة البيانات

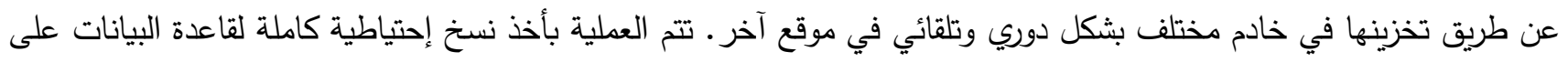
قرص صلب خارجي External Hard Disk (HDD) وتخزينها في مكان أكثر أماناً بشكل متكرر • يمكن استعادة هذه النسخة

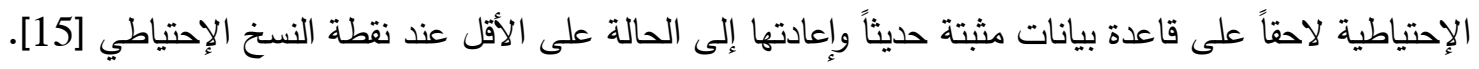

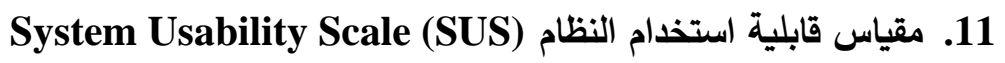
هي تقنية وخطة مدروسة وبسيطة تستخدم على نطاق واسع في تقييم تصميم النظام الذي تم تصميمهُ حديثاً على أسس إحصائية من خلال تتبع سلوك المستخدم والتعرف على انطباعاته، يتكون من استمارة إستبيان تحوي على عشرة جمل تم تطويرها

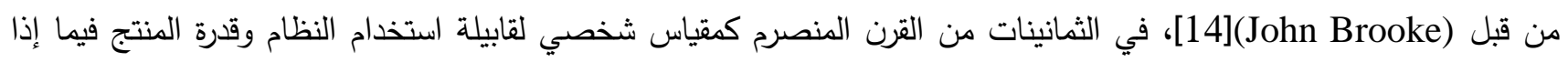
كان قادراً على تحقيق الغرض المقصود منهُ والاهداف المتطلبة والمأمولة [19]. 12. تحليل الهيكل التنظيمي وتحديد مستوى الصلاحيات ضمن النظام تعد المستويات الإدارية من الركائز الأساسية لنجاح العمل في المؤسسات، لذا تم دراسة وتحليل المستويات الإدارية

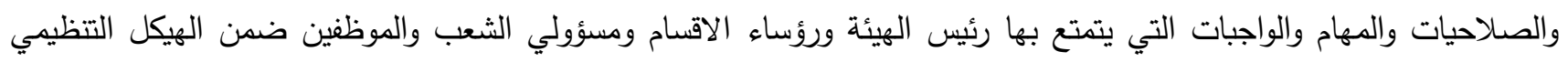
لهيئة استثمار نينوى وكما موضح بهرم مستويات الإدارة وصلاحيات الوصول إلى النظام وكما في الشكل (2): 


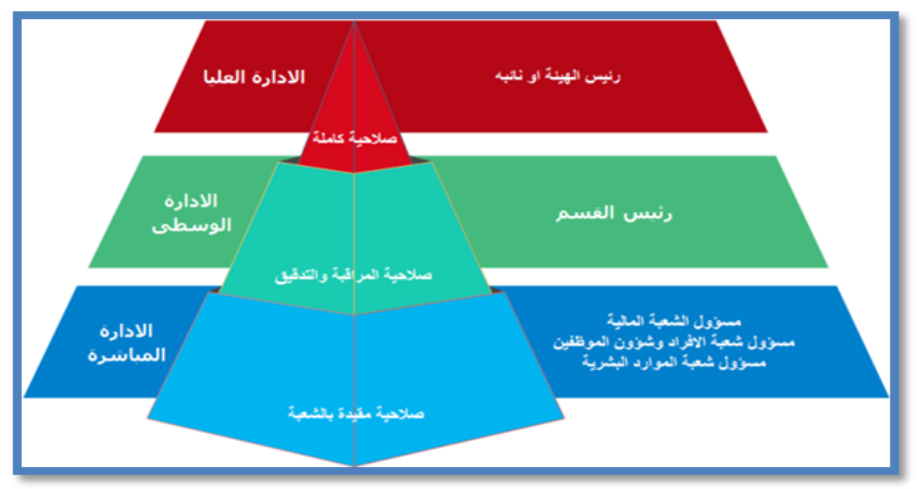

الثكل (2) هرم مستويات الإدارة وصلاحيات الوصول إلى النظام.

Data Flow Diagram (DFD) تحليل النظام باستخدام مخطط تدفق البيانات المستوى الأول من المخطط يسمى بالنموذج الاساس للنظام ويتكون من معالجة واحدة فقط تمثل النظام بأكمله، أما المستوى لئل

الثاني ويسمى النموذج المفصل لتوضيح العمليات التي ستجري في النظام بشكل أكثر تفصيلاً، وكما في الثكل (3):

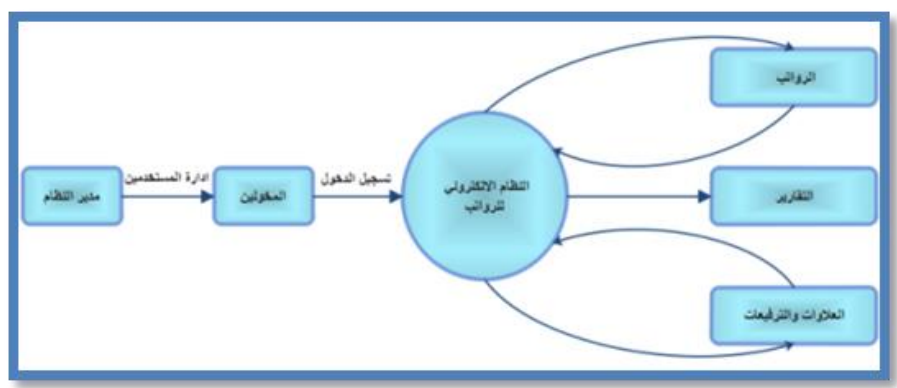

الثكل (3) النموذج الأساس لمخطط تدفق البيانات.

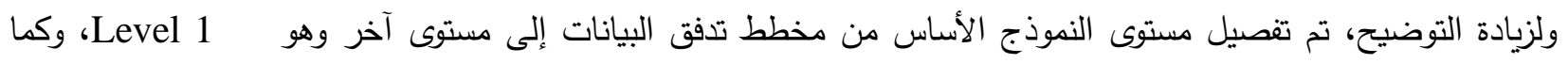

بالشكل (4):

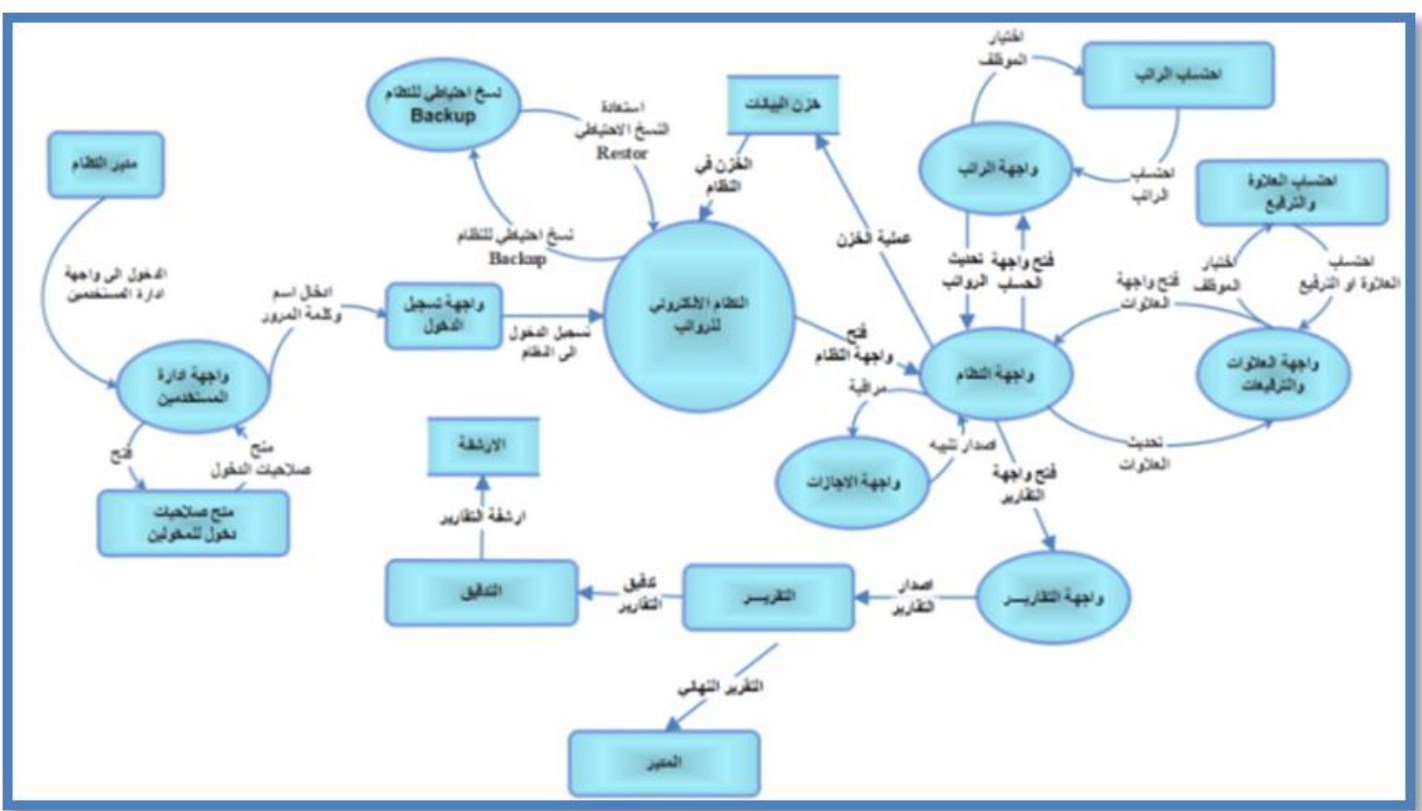

الشكل (4) المخطط الفقاعي لتدفق البيانات المستوى التفصيلي (1) Level 1. 
14. تحليل بيانات النظام باستخدام نموذج التواجد والعلاقة (Entity Relationship Diagram) تم تحليل قاعدة بيانات النظام المقترح باستخدام نموذج التواجد والعلاقة وتحديد التواجدات والصفات والعلاقات التي تربط بين تللك التواجدات كمكونات أساس، وسيتم التطرق إلى هذه القاعدة بالتفصيل. وكما في مخطط ER Diagram بالثكل (5) أدناه:

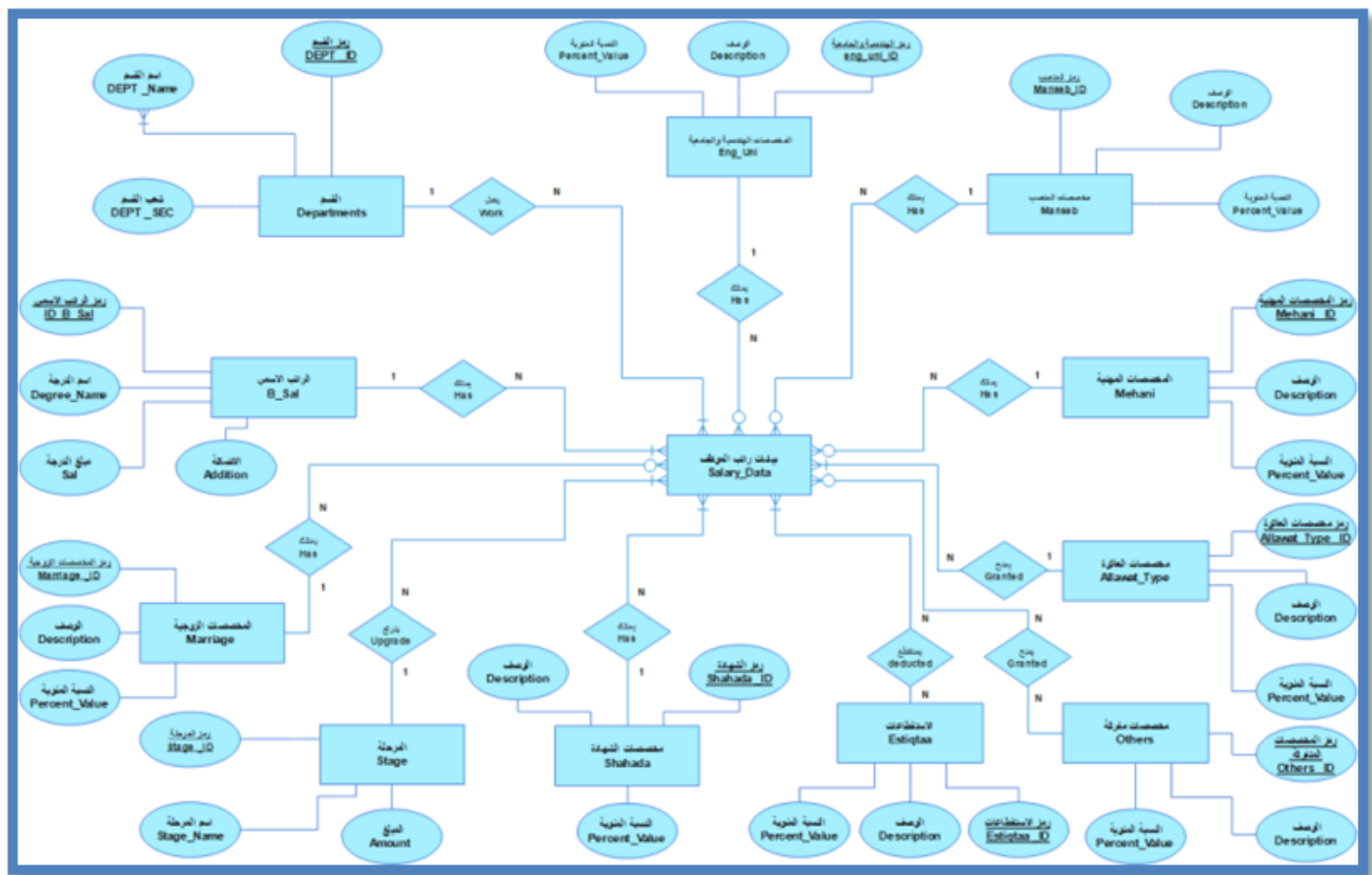

الثكل ER Diagram (5) Lموذج التواجدات والعلاقات.

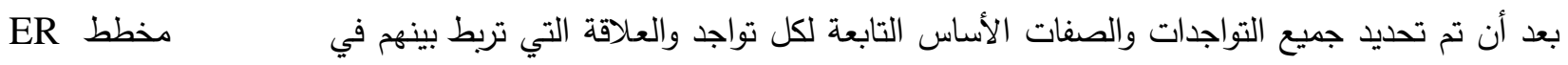

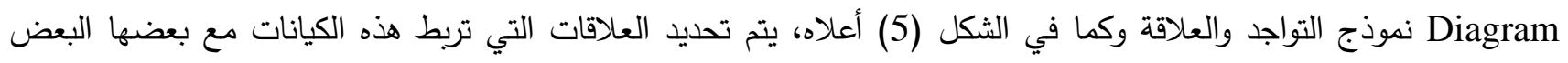
وكما يلي: • العلاقة بين تواجد بيانات راتب الموظف وتواجد الراتب الاسمي من نوع (واحد-لكثير)، إذ إن بيانات راتب الموظف يمتلك راتب اسمي واحد (إجباري مغرد). • العلاقة بين تواجد بيانات راتب الموظف وتواجد القسم من نوع (واحد-كثير)، إذ إن بيانات راتب الموظف يعمل في قسم واحد على الأقل (إجباري مفرد). • العلاقة بين تواجد بيانات راتب الموظف مع الع تواجد المخصصات الهندية والجامعية من نوع (واحد-كثير)، إذ يمتلك تواجد بيانات راتب الموظف على واحدة من هذه المخصصات (إختياري مفرد)، إذ يحتوي تواجد المخصصات الهندسية والجامعية على رئى عدة مخصصات. • العلاقة بين تواجد بيانات الراتب مع تواجد مخصصات المنصب من نوع (واحد-لكثير)، إذ يمتلك تواجد بيانات راتب الموظف

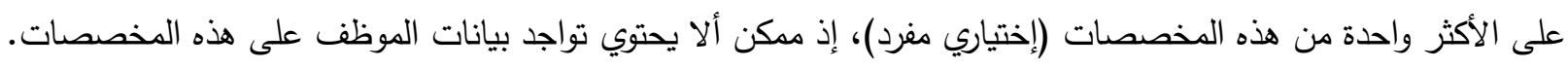

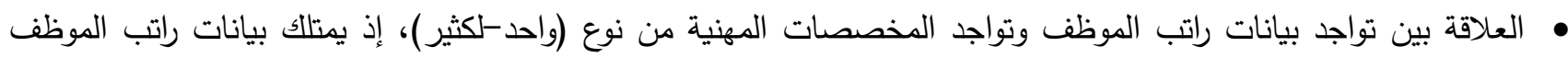
على الأكثر هذه المخصصات (إختياري مفرد). • العلاقة بين تواجد بيانات راتب الموظف وتواجد مخصصات العلاوة من نوع (واحد-لكثير)، إذ يمنح للموظف على الأقل مخصصات علاوة (إجباري مفرد). 
• العلاقة بين تواجد بيانات راتب الموظف وتواجد مخصصات متفرقة من نوع (كثير -كثير)، إذ يمنح على الأقل أكثر من واحد من هذه المخصصات (إجباري متعدد). • العلاقة بين تواجد بيانات راتب الموظف وتواجد الاستقطاعات من نوع (كثير -كثير)، إذ يستقطع على الأقل أكثر من واحدة من هذه الاستقطاعات (إجباري متعدد). • العلاقة بين تواجد بيانات راتب الموظف وتواجد مخصصات الاتعدد) الشهادة (واحد-كثير ). • العلاقة بين تواجد بيانات راتب الموظف وتواجد المرحلة من نوع (واحد-لكثير)، إذ يمتلك تواجد بيانات راتب الموظف على ترفيع واحد على الأكثر (إجباري مفرد). • العلاقة بين تواجد بيانات راتب الموظف وتواجد المخصصئ الات الزوجية من نوع (واحد-كثثر)، إذ ممكن أن يمتلك تواجد بيانات

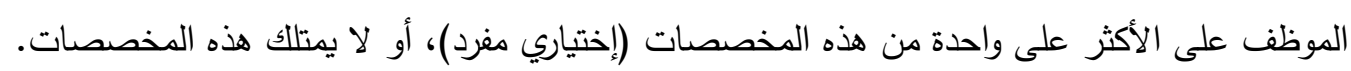
تتضمن قاعدة بيانات النظام الإكتروني للرواتب، الموضحة في الثكل (6) نموذج العلاقات بين التواجدات في النظام

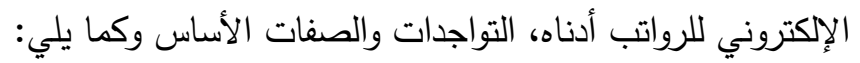

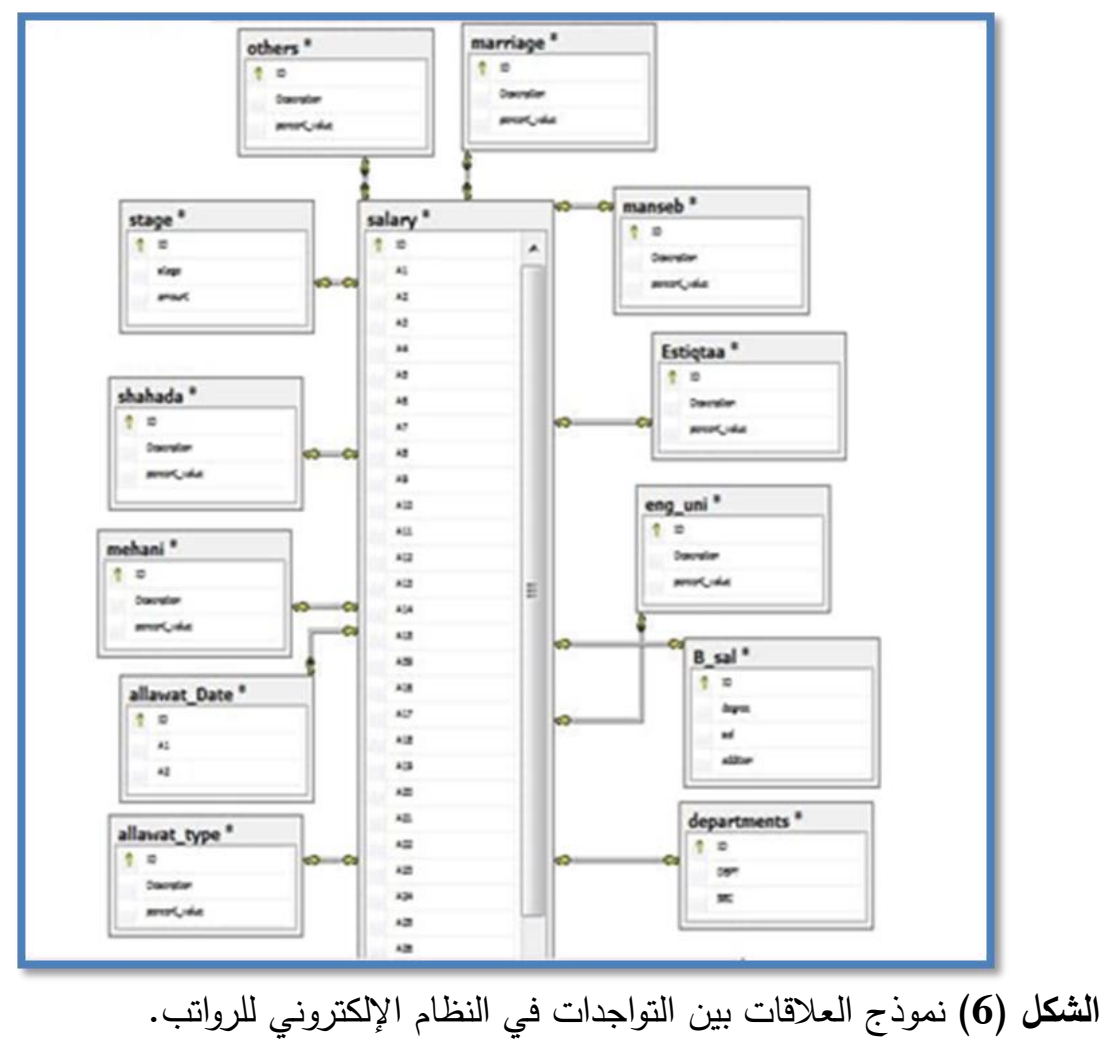

15. مراحل حياة النظام

إن عملية تشغيل النظام تتلخص بعدة مراحل كما في الشكل (7) والتي تتضمن بالتالي:

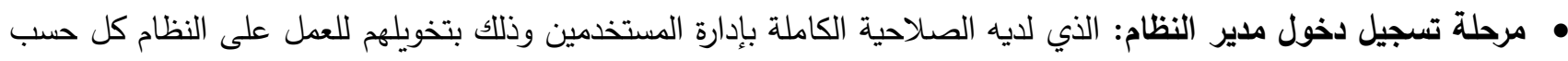

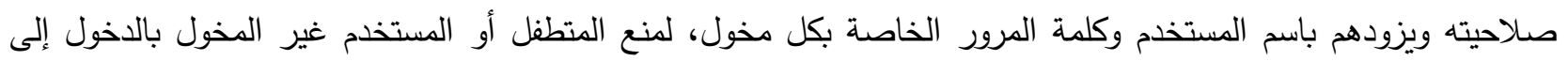
النظام، ويتم تسجيل الدخول للمخولين بعد حصولهم على مفتاح الاخول.

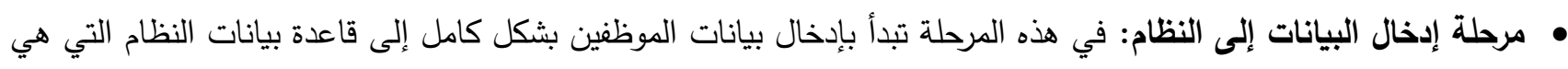
ستكون أساساً للعمل عليها من قبل الموظفين المخولين باستخدام النظام، والتي كانت بيانات ورقية في النظام التقليدي، إذ إذات ستصبح في هذه المرحلة إلكترونياً وستخزن في هذه القاعدة كخزن مؤقت سيتم تعديلها في المراحل اللاحقة. 
• الخزن في قاعدة البيانات: في هذه المرحلة البيانات جاهزة للتعديل وهي أي تغيير على الراتب أو على بيانات الموظفين أو أي

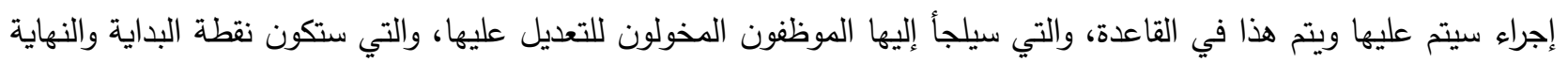
لكراحل احتساب الرواتب والعلاوات والترفيعات. • مرحلة إضافة العلاوات والترفيعات للمستحقين: في هذه المرحلة يقوم الموظف المختص بشعبتي الأفراد والموارد البشرية

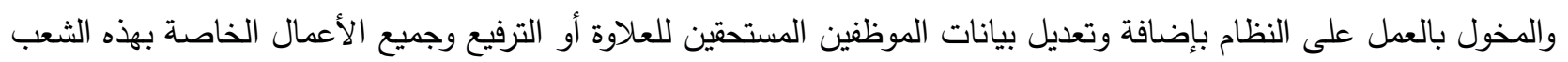
وتعديلها في قاعدة البيانات. • مرحلة تعديل البيانات المالية: في هذه المرحلة يقوم الموظف المختص بالثعبة المالية بتحديث البيانات المالية للموظفين الذين

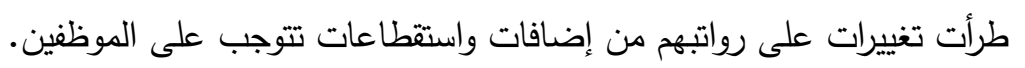
• مرحلة احتساب الراتب: في هذه المرحلة يتم احتساب جميع الرواتب التي تم التعديل عليها من إضافات واستقطاعات وجعلها

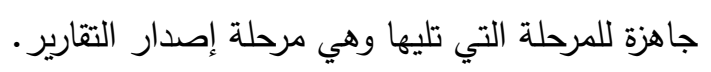
• مرحلة إصدار التقارير: في هذه المرحلة يتم إصدار التقارير التي تحوي على جميع البيانات المالية للموظفين في المؤسسة وكذلك بياناتهم الإدارية لتكون جاهزة للمرحلة التي تليها وهي مرحلة التدقيق. • مرحلة التدقيق: وهي مرحلة تدقيق جميع البيانات من قبل الموظف المختص والمخول للعمل على هذا النظام لتكون جاهزة

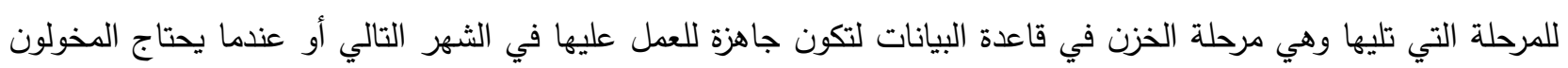
إجراء تغييرات على البيانات.

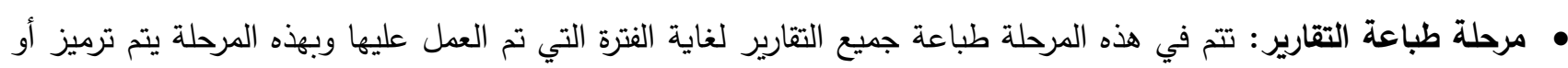
تسمية التقارير مثلا تسمى تبعاً للشهر الذي تم تعديل البيانات لتكون جاهزة وقابلة للأرشفة.

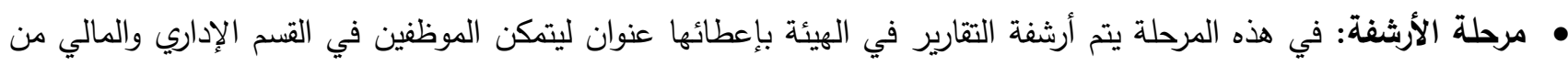

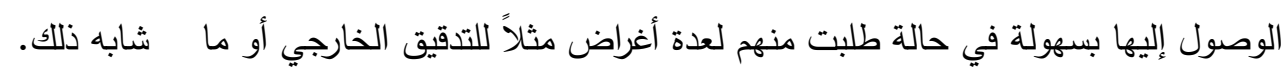

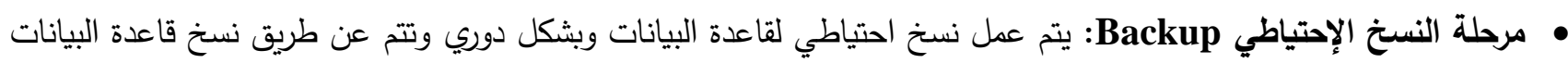

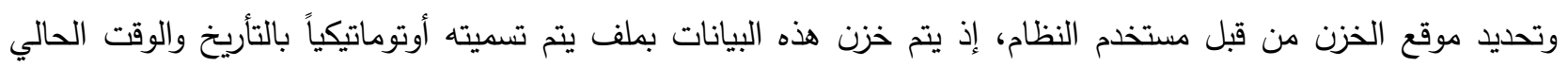

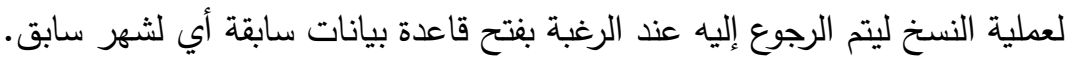

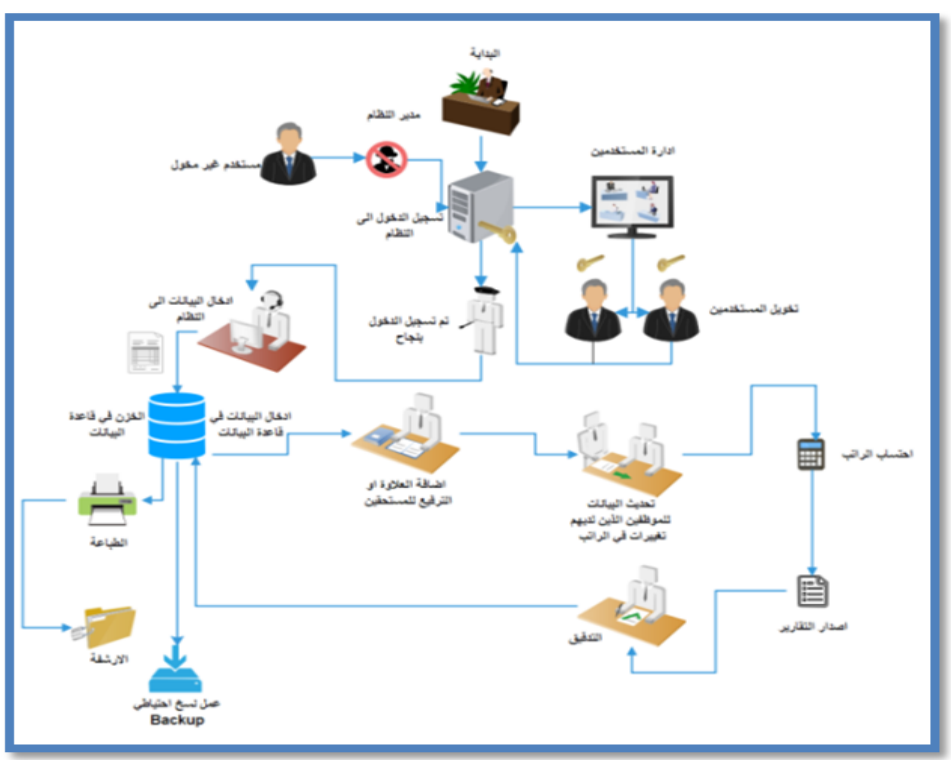

الثكل (7) مراحل حياة النظام. 
16. تأسيس شبكة محلية لاسلكية لتطبيق النظام الإلكتروني للرواتب

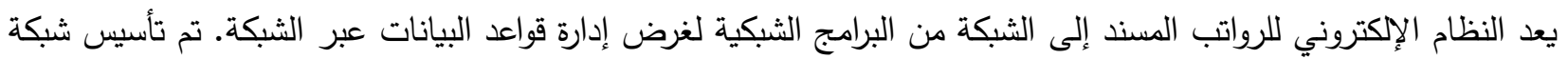
محلية لاسلكية مع مراعاة ثلاث نقاط أساس هي حجم الشبكة Size، أسلوب توصيل الشبكة Model، نمط توصيل الشبكة Typology

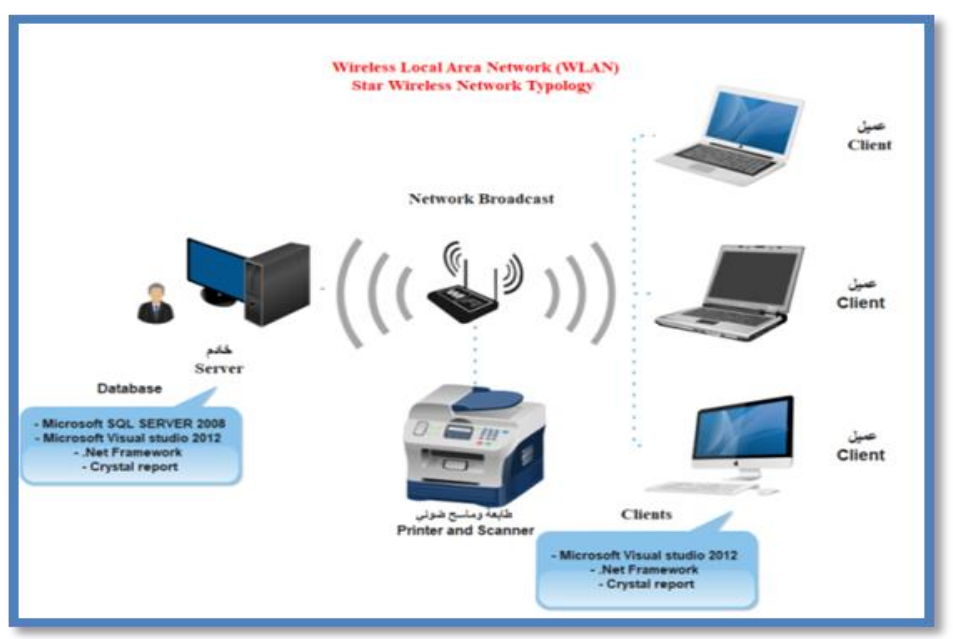

الثكل (8) معمارية شبكة النظام الإكتروني للرواتب [21].

1 الأجهزة المستخدمة لإنشاء الشبكة المحلية:

• جهاز راوتر لاسلكي (Wireless Router).

• اسوب رئيس يعمل كخادم (Server).

• جهاز حاسوب محمول (Laptop) يعمل كعميل (Client).

• جهاز طابعة ملونة (Printer).

2.16 تهيئة النظام للعمل على الثبكة المحلية:

يتم تثبيت النظام الاكتروني للرواتب على جميع الحواسيب في الشبكة والتي تتكون من الحاسوب الرئيس (Server) وحواسيب

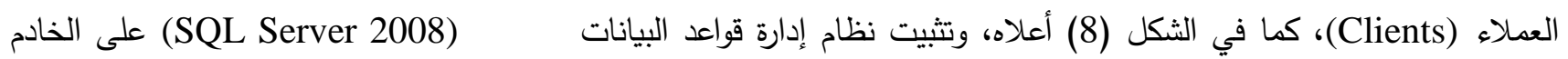
(Microsoft الرئيس فقط لأنه يحتوي على قاعدة البيانات المركزية، وكذلك يتم تثبيت في جميع الحواسيب على الثبكة برنامج (Crystal Report) و لتوليد التقارير . (NET Framework) Visual Studio 2012) يتميز النظام الإكتروني للرواتب بمستويات أمان وهي: • أمان الثبكة اللاسلكية: من خلال ضبط إعدادات الحماية بجهاز الراوتر الذي يربط الثبكة اللاسلكية من المستخدمين غير المخولين للدخول إلى الشبكة من خلال تشفير الدخول إلى الشبكة اللاسلكية أو تحديد الحواسيب التي يمكن لها الدخول إلى الثبكة باستخدام خاصية (MAC) الخاصة بكل حاسوب أو من خلال تغيير اسم الشبكة (SSID) أو وضع رمز مرور سري أو إخفاء اسم الشبكة. لتأمين وصول جميع المستخدمين المخولين إلى الشبكة ومن ثم إلى النظام وعلى وفق الصلاحيات الممنوحة من مدير النظام وذلك عن طريق ادخال اسم مستخدم ورمز المرور الخاص بالمخول. • أمان نظام إدارة قواعد البيانات: إذ يقوم بتقديم العديد من المميزات التي تم تصميمها لتحسين أمن بيئة قاعدة البيانات من خلال التثفير والموثوقية (المصادقة) إذ يوفر (SQL Server 2008) نوعين من المصادة إندة: للاتصال مع خادم قاعدة البيانات، والذي تم تطبيقه في هذا المشروع. 
يبدأ تشغيل النظام مباشرة بالضغط على ايقونة البرنامج المتواجدة على حاسبة الخادم أو على حاسبات العميل بالضغط عليها

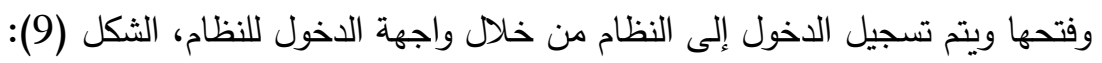

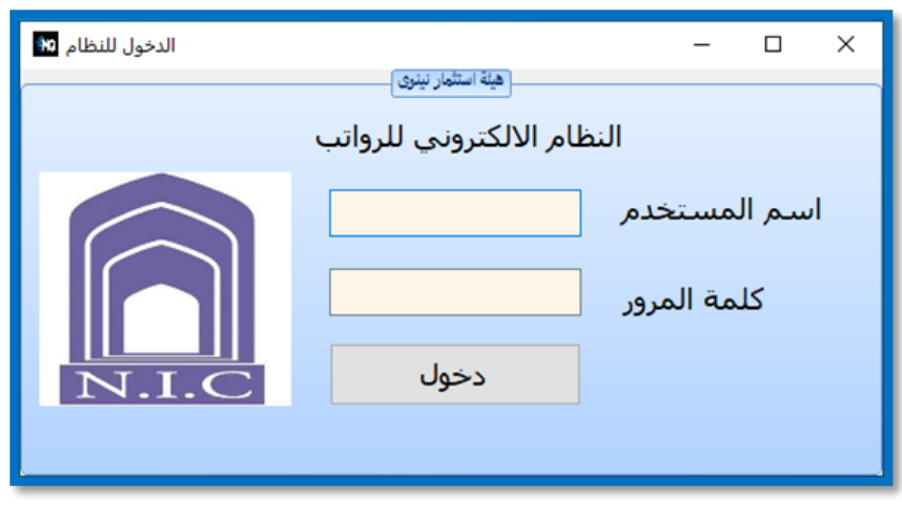

الشكل (9) واجهة الدخول إلى النظام.

بعد عملية الدخول إلى النظام عن طريق اسم المستخدم ورمز المرور الصالحين سوف يتم الانتقال إلى واجهة بيانات النظام

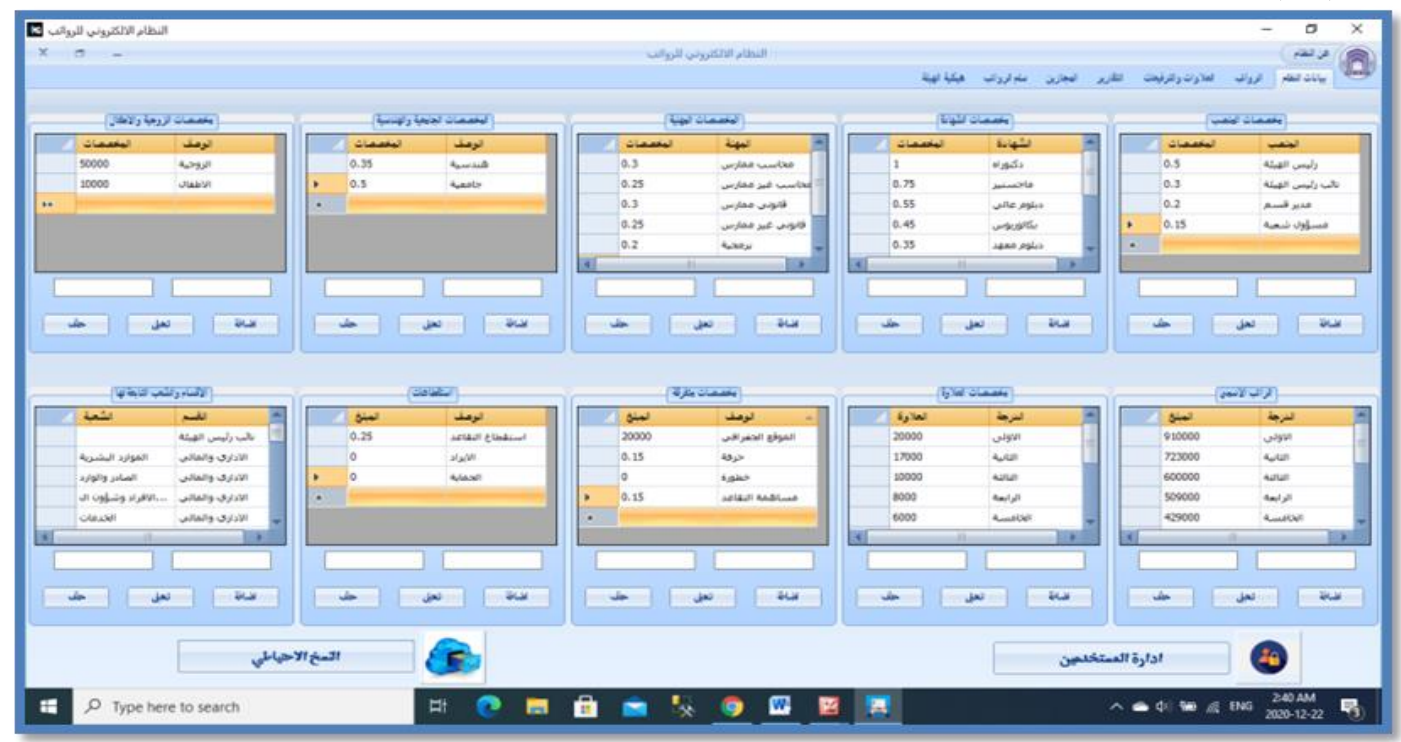

الثكل (10) واجهة بيانات النظام الأساسية.

18. واجهة بيانات النظام الرئيسة

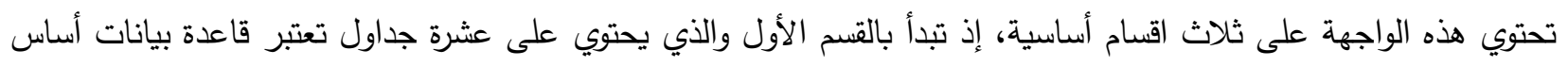

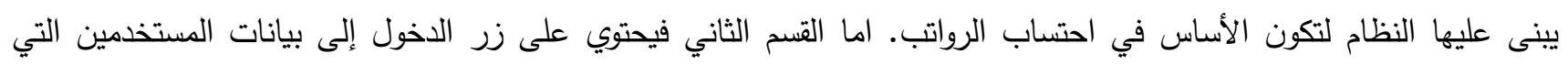

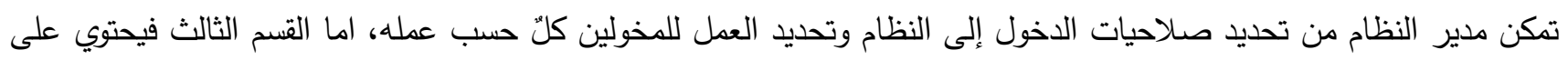
زر النسخ الإحتياطي Backup الذي من خلال الضغط عليه سيتم الانتقال إلى واجهة النسخ الإحتياطي ومن خلاله سيتم عمل نسخ لبيانات النظام وكذلك إمكانية عمل استرجاع Restore لهذه البيانات عن الحاجة اليها. وتكون هذه الواجهة من النوع المفتوح أي ممكن تحديث بيانات الجداول حسب احتياجات المؤسسة التي ترغب بتطبيق هذا النظام عن طريق الإضافة والحذف والتعديل، اذ يتم تقسيم العمل فقط في القسم الإداري والمالي للمؤسسة وكما سيتم توضيحها في واجهة الرواتب وواجهة العلاوات والترفيعات. 
تتكون واجهة الرواتب من أربعة اقسام أساسية والموضحة بالثكل (11) أدناه، إذ يحتوي القسم الأول على بيانات الموظفين

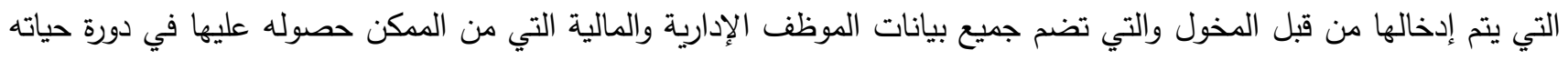
الوظيفية، اما القسم الثاني من واجهة الرواتب قسم احتساب الراتب والتي يتم من خلالها احتساب مفردات راتب الموظف بلاتبات الاعتماد

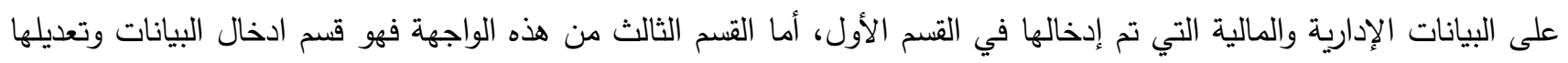

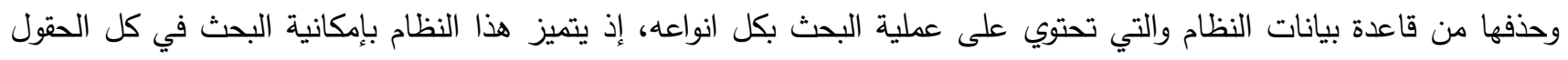
وعلى أي بيانات يحتاج الوصول اليها بكل سهولة، كما تحتوي على ازرار إضافة بيانات الموظف وحذفها وتعديلها، كما يحتوي هذا

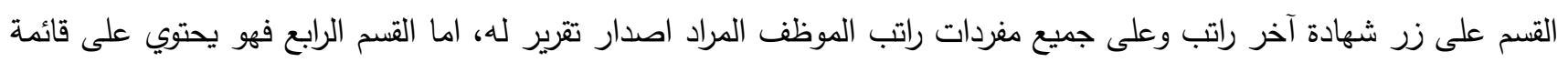

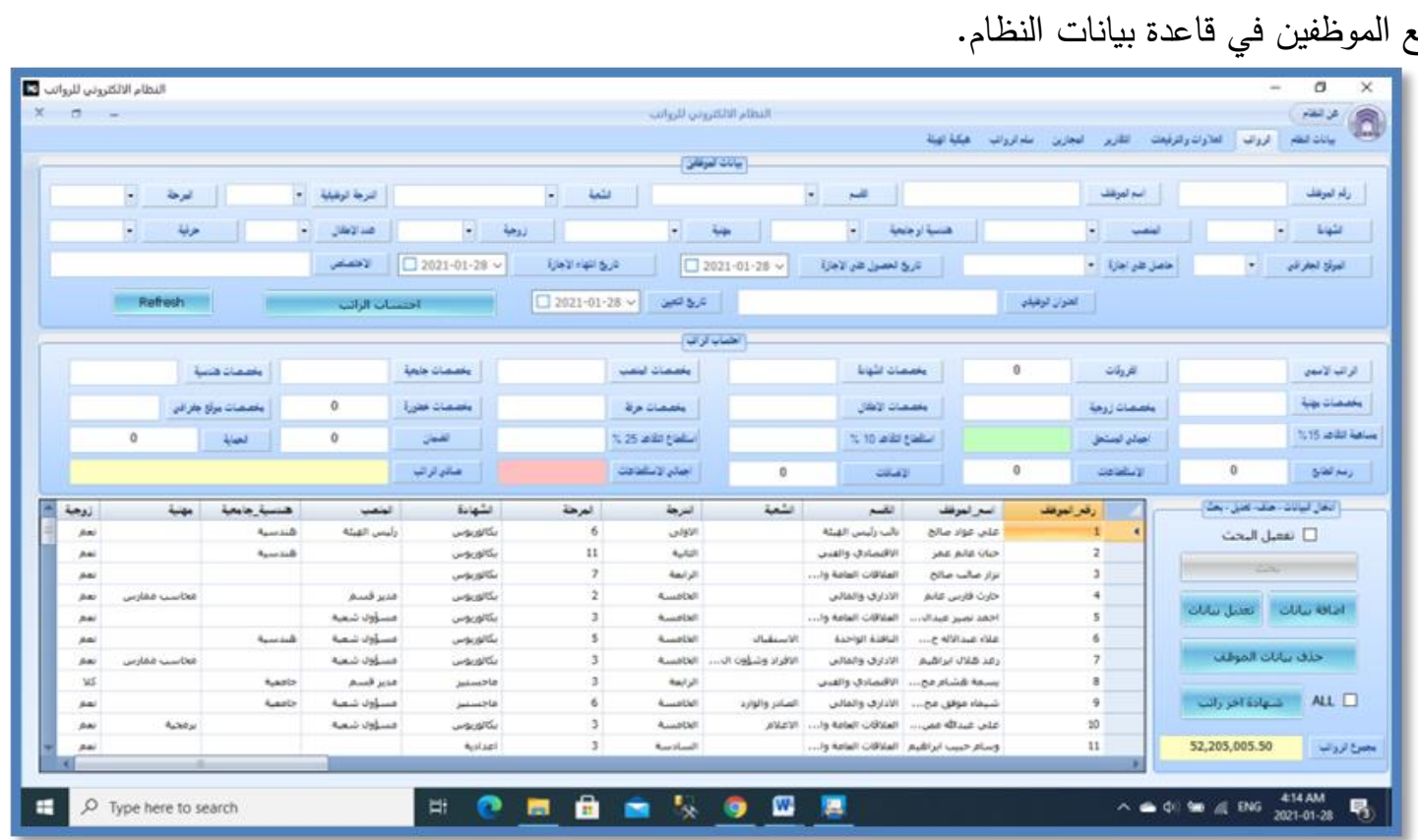

الشكل (11) واجهة الرواتب.

20 . واجهة العلاوات والترفيعات

تضم واجهة العلاوات والترفيعات على خمسة اقسام، القسم الأول هو قسم آخر علاوة أو ترفيع والذي يتم إضافة وتعديل وحذف إتف اسم الموظف وتأريخ آخر علاوة والدرجة والمرحلة، اما القسم الثاني وهو قسم كتب الثكر والعقوبات والذي يتم من خلاتله الدات ادخال

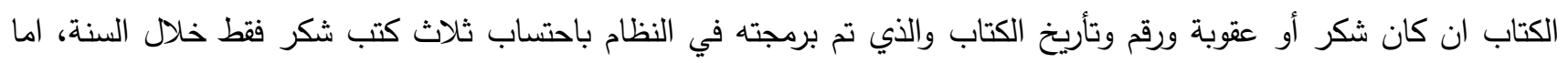

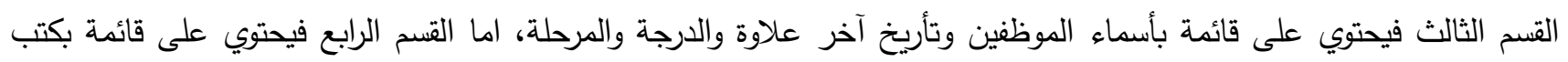

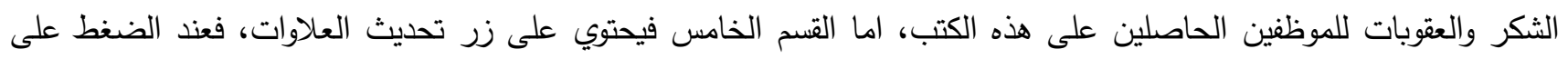
هذا الزر سيتم تحديد الموظفين المستحقين للعلاوة أو الترفيع بتتبيه عن طريق تظليل حقل الموظف باللون الغامق وكما في الثكل أدناه 


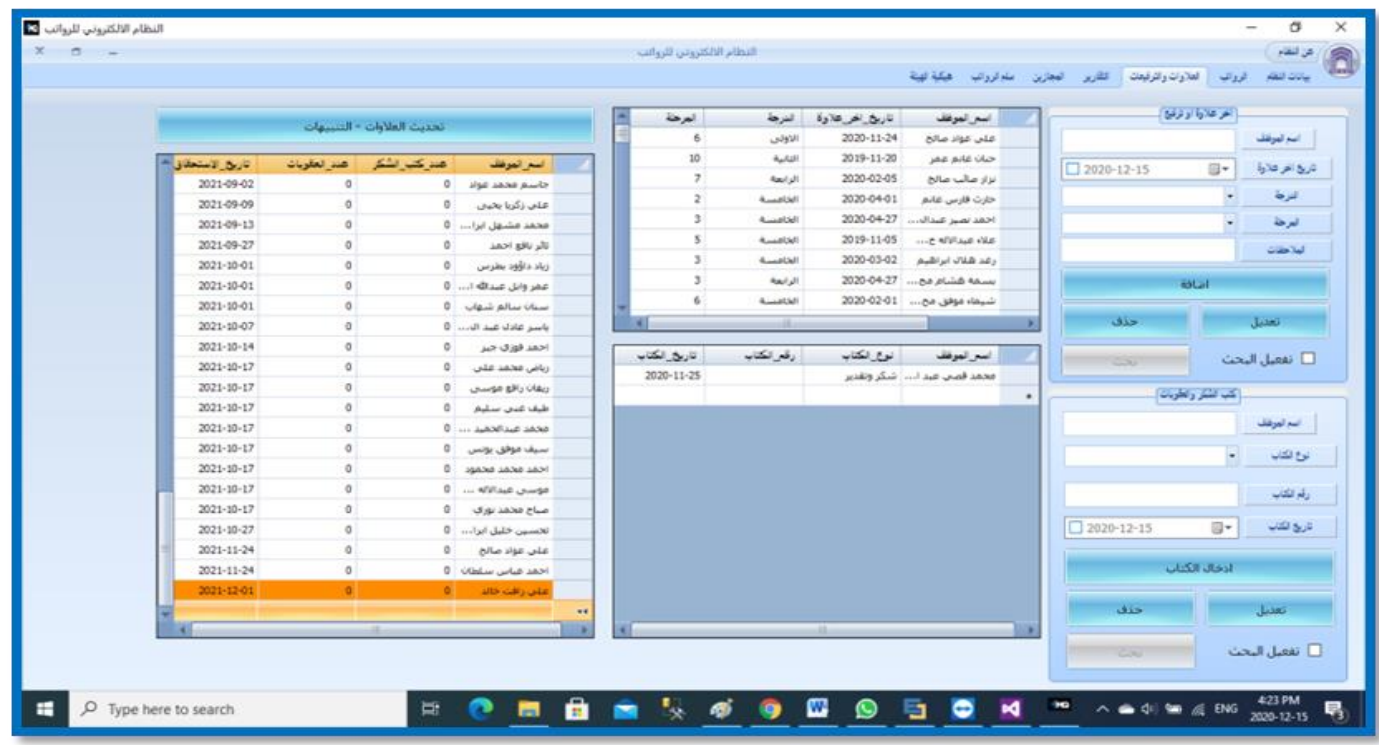

$$
\text { الثكل (12) واجهة العلاوات والترفيعات. }
$$

21. واجهة التقارير

تعتبر واجهة التقارير من الاعمال الأساسية في اصدار قوائم الراتب، والتي يُعتمد عليها في صرف الرواتب والتي يتم تدقيقها

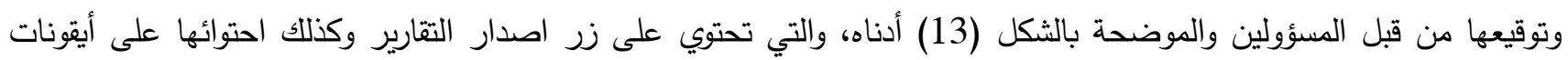
مختلفة منها أيقونة تصدير التقارير والتي تعمل على تصدير التقارير وخزنها واختيار نوع الخزن، أيقونة طباعة لطباعة التقارير،

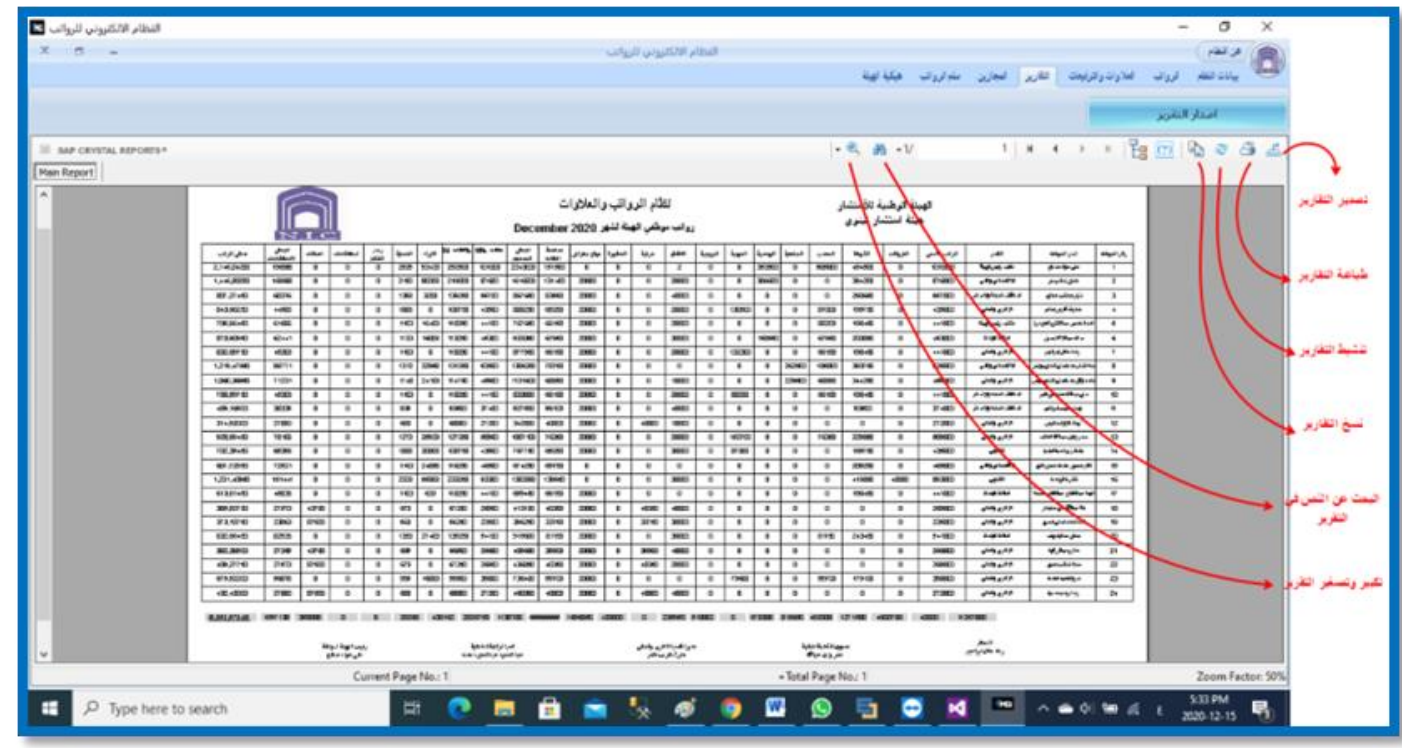

وغيرها.

الشكل (13) واجهة التقارير.

22.

صمدت هذه الواجهة لتساعد شعبة الافراد وشؤون الموظفين لإدارة المجازين بجميع انوع الاجازات المتمتعين بها لاحتوائها على

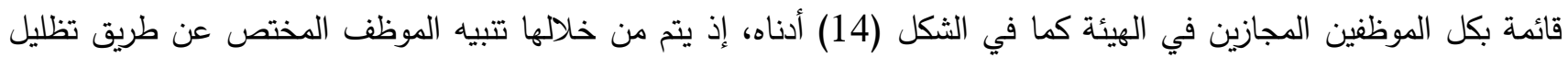
الحقل باللون الغامق للموظف المنتهية اجازته والتي تم تحديد نوع الاجازة وتأريخ الحصول على الاجازة وتحديد تأريخ انتهاءها في واجهة الرواتب. 


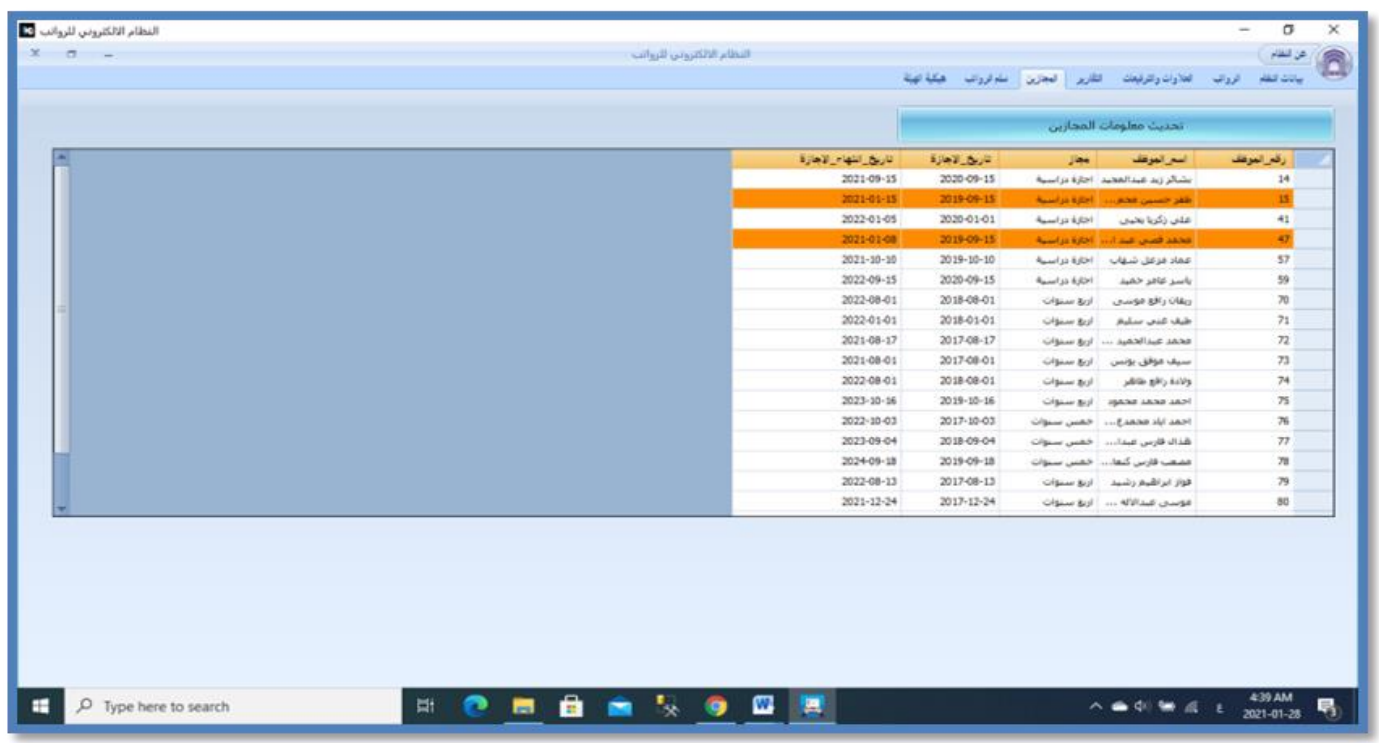

الثكل (14) واجهة الاجازات.

23. إختبار قابلية استخدام النظام

تم اختبار النظام بواسطة تقنية مقياس قابلية النظام على الموظفين في القسم الإداري والمالي في الهيئة والذين يعملون على التى

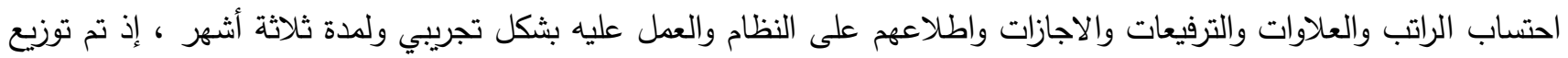

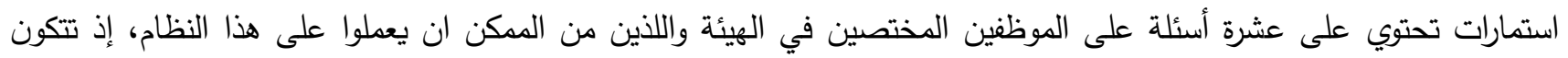
استمارة الاستبيان من خمسة أسئلة إيجابية واخرى سلبية، ووزعت على (25) موظف في هيئة استثمار نينوى اللذين اطلعوا على لئى النظام واللذين يمكنهم العمل على هذا النظام في المستقبل، وتم ملئ واسترجاع هذه الاستمارات، والموضحة بالثكل (15) (15) أدناه، ليتم

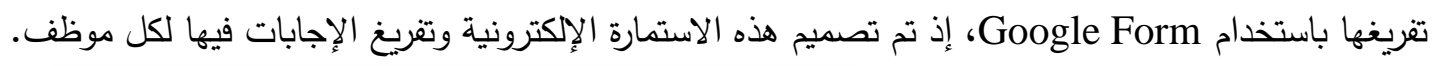

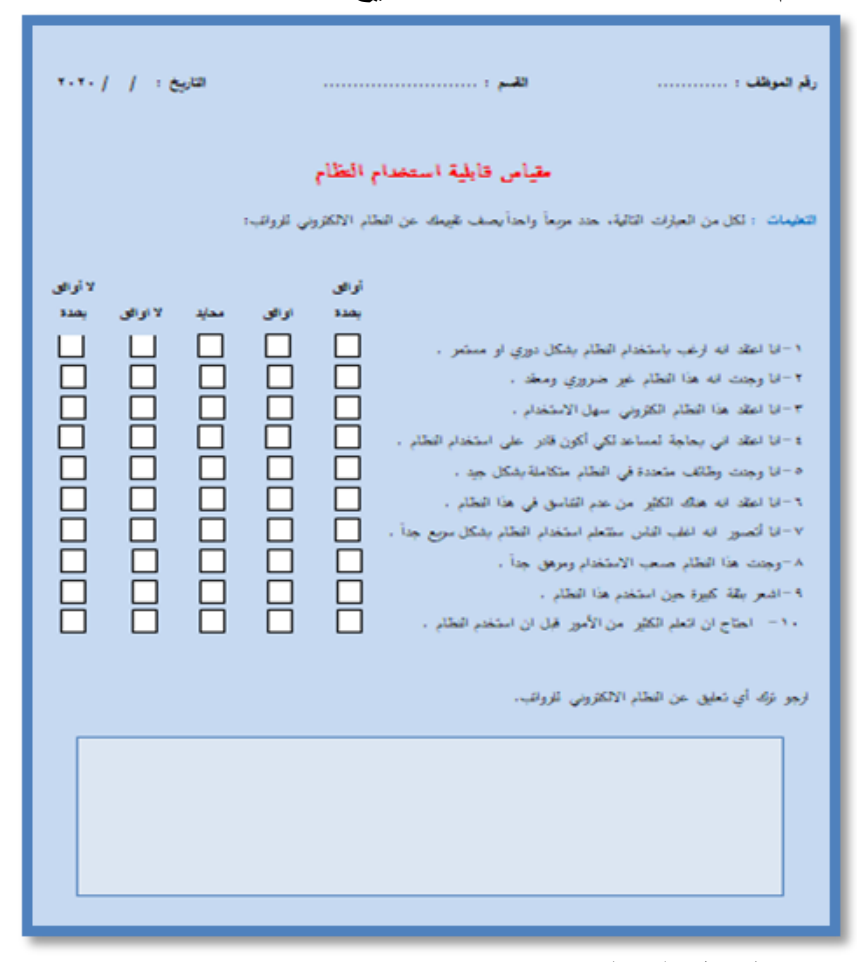

$$
\text { الشكل (15) استمارة الاستبيان. }
$$


من خلال انجاز هذا البحث تم التوصل إلى عدد من الاستتتاجات والتي تمخضت عنها وتتلخص بالتالي: 1. احتساب الرواتب الكترونياً من خلال تحديد خدمة الموظف والثهادة الحاصل عليها والمخصصات الثابتة والمتغيرة والاستقطاعات وغيرها من الاعمال المحاسبية بطريقة سلسة وسرعة بالمقارنة مع النظام التقليدي المستخدم في الهيئة، مع تجهيز كثف الرواتب للموظفين. 2. موثوقية العمل على النظام إذ انه قلل من مسؤولية الموظف المختص العامل في الثعبة المالية من حيث الاعتماد على بيانات أساسية ومعادلات حسابية دقيقة تم برمجتها وإدخالها في قاعدة بيانات النظام تقوم باحتساب جميع مغردات الراتب بصورة دقية التيقة وخالية من الأخطاء بدون الرجوع إلى أساليب النظام التقليدي. 3. تحديد منح الصلاحية للدخول إلى النظام من خلال اسم المستخدم وكلمة المرور للموظف المخول هذا ما جعل النظام أكثر أمنا وسرية وخصوصية في عملية الوصول إلى بيانات الموظفين إذ تم تقسيم الصلاحيات على ثلاث مستويات تحدد مستوى الإدارة التي يعمل فيها الموظف ودخوله للواجهة المخصصة له والتعامل مع البيانات المسموح له برؤيتها فقط. 4. حفظ بيانات الموظفين في قاعدة بيانات واحدة ضمن نظام (MS SQL Server 2008) التي توفر حماية للبيانات من الفقد.

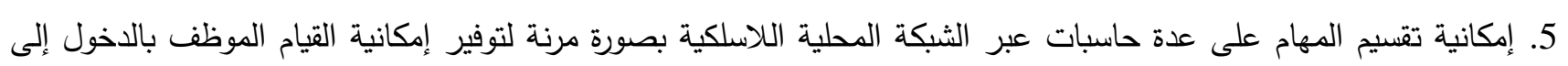
الواجهة الخاصة به والقيام بعمله والربط ما بين شعب قعم الإداري والمالي في الهيئة. 6. توفير الجهد والوقت والدقة والسرعة وسهولة الاستعلام عن بيانات منتسب محدد بمجرد تفعيل زر البحث سيتم استدعاء كافة بيانات المنتسب وطباعتها مع إمكانية البحث في كافة الحقول الموجودة.

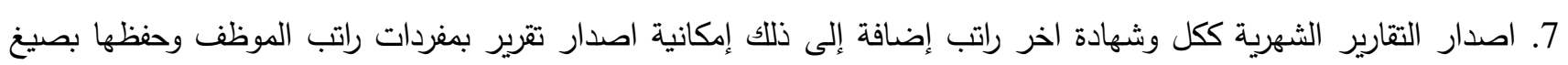
إلكترونية متعددة مثل (Word, Excel, PDF, CSV, CRT, RTF, XML). 8. تتبيه الموظف الاداري المختص المسؤول عن اجازات الموظفين لتاريخ انتهاء الاجازة التي استحصل عليها الموظف عن

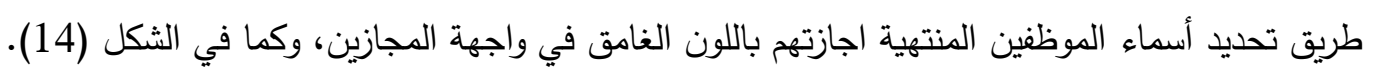
9. تتبيه الموظف الإداري المختص لتاريخ استحقاق الموظفين للعلاوة السنوية في كل شهر واصدار تقرير عن المستحقين للعلاوة عن طريق تحديد أسماء الموظفين المستحقين للترفيع او العلاوة باللون الغامق في واجهة العلاوات والترفيعات، وكما في الشكل

10. نسخ احتياطي لقاعدة البيانات الخاصة بالهيئة للدحافظة عليها من التلف وارشفتها والعودة اليها عند الحاجة بوضع النسخة في مجلد ثانٍ ضمن نفس جهاز الحاسوب أو نقلها إلى جهاز خزن خارجي أو عمل خزن سحابي (Cloud Storage) في حالة يوجد للهيئة حساب خادم خاص بها. 11. نفذ النظام واختبر على بيانات حقيقة لموظفي الهيئة تمثلت بـ(81) موظف ولمدة ثلاثة أشهر تم احتساب رواتبهم وتم اصدار التقارير لهم وتدقيقها، إذ تبين إمكانية العمل على هذا النظام وسهولة استخدامه ودقة الكشوفات التي تم إصدارها للأشهر تشرين الأول وتشرين الثاني وكانون الأول لعام 2020.

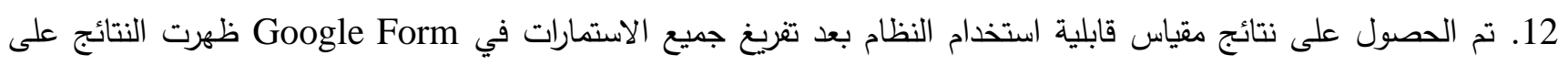

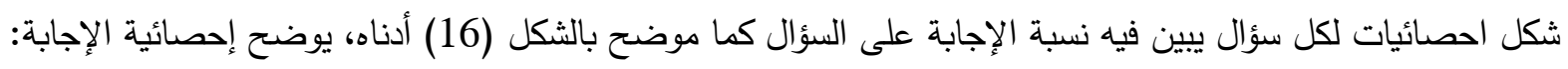




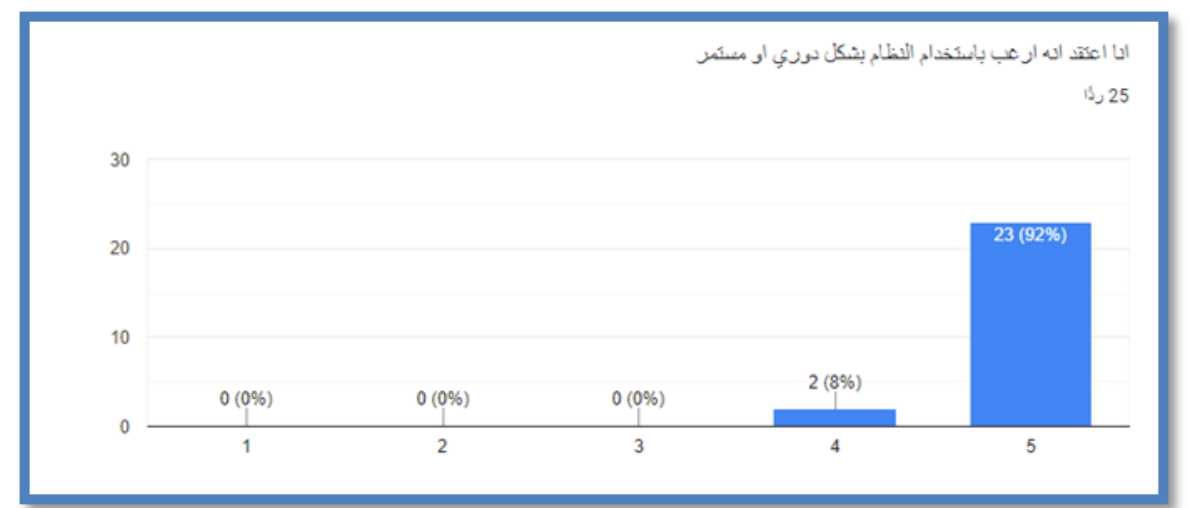

$$
\text { الشكل (16) إحصائية استبيان السؤال الأول. }
$$

توضح الإحصائية بأن نسبة الإجابة على السؤال الأول كانت (92\%) وهي حصيلة إجابة (23) موظف اختار الإجابة رقم (5) والتي تعني (أوافق بثدة)، والاجابة رقم (4) والتي تعني (أوافق) توضح نسبة الإجابة (8\%) والتي اختيرت من قبل موظفين الإنين التين

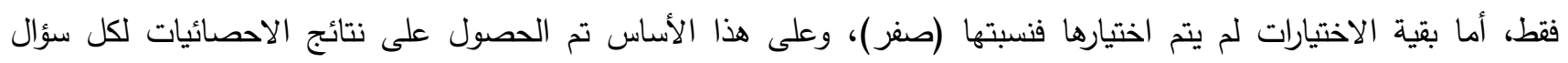

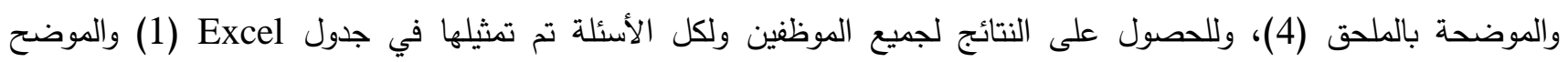
بالتقصيل أدناه:

$$
\text { الجدول (1) معدل نتائج الاستبيان. }
$$

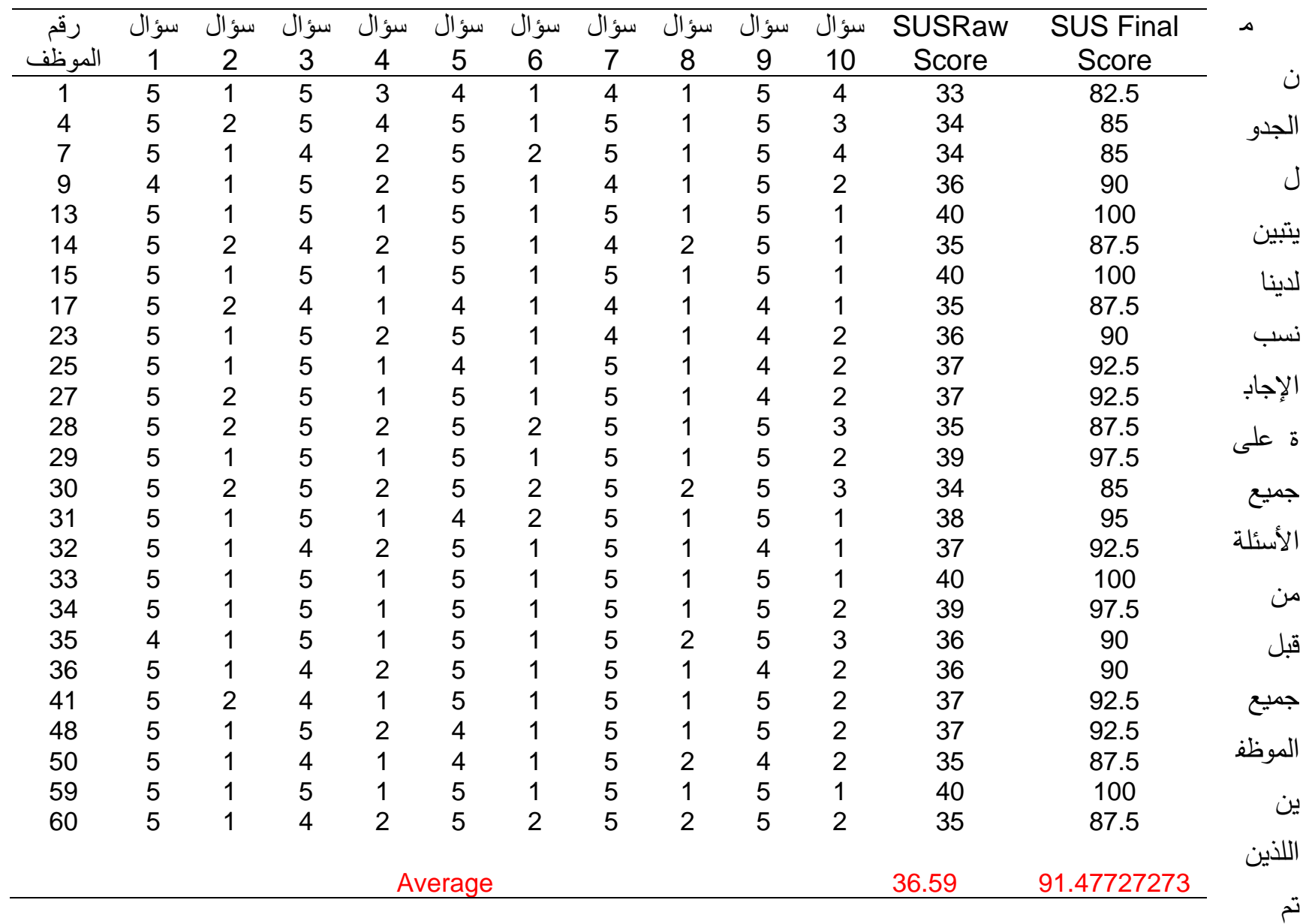

توزيع استمارة الاستبيان عليهم، إذ يحتوي الجدول على رقم الموظف وما يقابله نتيجة جوابه على السؤال المطروح، فتم الإجابة على هذه الأسئلة بما يراه الموظف من قابلية استخدامه للنظام وجمعت النتائج عن طريق معادلة خاصة والتي تم ذكرها في الفصل الثاني 


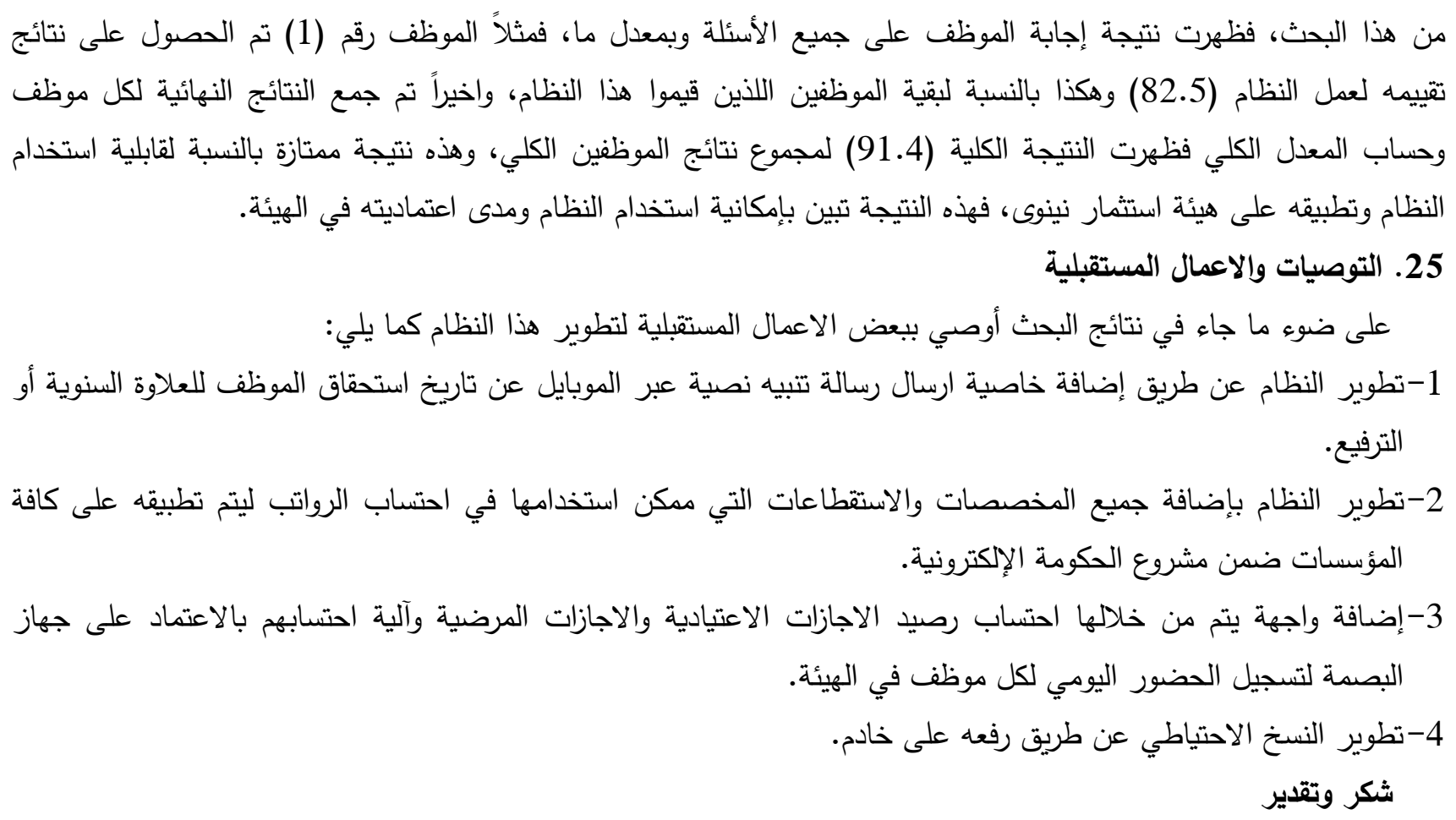

يتقدم الباحثون بالثكر لقسم علوم الحاسوب، كلية علوم الحاسوب والرياضيات، جامعة الموصل وهيئة استثمار نينوى.

المصادر

[1] Majeed, F A, Ali, A A H, (2019), The Influence of Uniard management has been explored in achieving the performance management approach, Karbala University, Warith Journal of Scientific Research, VOL (1), No (1), p 2618- 0278.

[2] Mahal, K A N., (2019), Design an electronic system for bonus and promotions a model proposed at some colle of the university of Mosul, higher Deploma thesis, Mosul, college administration and economic, university of Mosul.

[3] Al-Jamal, N., (2019), Definition of Electronic Accounting, Row electronic magazine, 5-1.

[4] Laudon K C., \& Laudon J P., (2018), Management Information Systems Managing the Digital Firm, (3rded) Essex: Pearson Education Limited, P (50).

[5] Al-Khalil, A A H., 2018, Human resource management, Master thesis, Damascus: Syrian Virtual University, P (20).

[6] Rozario M A D., (2018), Project On Employee Database and Payroll Management System, Master thesis, Kolkata: Rcc Institute of Information Technology, Maulana Abul Azad University of Technology, P (1)

[7] Abd Al-Aziz,W A., (2017), The effect of implementing the government financial information management system on the efficiency of public financial management system in Egypt, Master thesis, Cairo: The national Institute.

[8] Olawale K E., (2017), Design and Implementation of Employee Information and Payroll System, Bachlor thesis, (Using Caleb University as A Case Study), Imota Lagos: College of Pure and Applied Science caleb University, P (9).

[9] Adeoye A O., Atiku S O., \& Fields Z., (October, 2016), Structural Determinants of Job Satisfaction: The Mutual Influences of Compensation Management and Employees' Motivation, Journal of Economics and Behavioral Studies, 8(5), P 27-38.

[10] Mahajan K., Shukla S., \& Soni N., (January, 2015), A Review of Computerized Payroll System. International Journal of Advanced Research in Computer and Communication Engineering, 4(1), P 67-80. doi: 10.17148/IJARCCE.2015.4113 
[11] Al-Asaadi, M T., (2015), Database using Visual FoxPro, P (3)., Mosul: The department of computer system- The Technical Institute,

[12] Thabit, H T., (2014), The requirements of transformation from traditional accounting to the electronic accounting, P (2), the first scientific conference of Administrative Technical college, Mosul: Technical college.

[13] LIM F P C., (2013), Impact of Information Technology on Accounting Systems, Asia-Pasific Journal of Multimedia Services Convergent with Art, Humanities and Sociology, 3(2), P 93-106.

[14] Peres S C., Pham T., \& Phillips R., (2013), Validation of The System Usability Scale (SUS): SUS IN THE WILD. the Human Factors and Ergonomics Society Annual Meeting, SAGE, 57(1), P 192-196.

[15] Putra Z, Yuliana S, Waisen A, Benny, (2020), Data Management System for Thesis Monitoring at STMIK IBBI Using B-Model, 3rd International conference on mechanical, Electronics, Computer, and Industrial Technology (MECnIT), P (5), Medan, Indonesia.

[16] Por, L Y, Ong, S Y, Beh D, Ismail, M, (2010), Faculty of Computer Science and Information Technology, university of Malaya, Malaysia, The International Arab Journal of Information Technology, Vol. 9, No. 4.

[17] Hasan M R., Paul R., \& Nur-E-Alam M D., (2012), Design and Development of Payroll Management System, Dhaka: The People's University of Bangladesh (PUB), Department of Computer Science and Engineering.

[18] Zuta M. K., (2012), E-Leave of Human Resource Management Office Leave Management System Payroll Management System Review of related literature A System for Payroll Management 1. Delhi: Department of Biostatistics, Defense Institute of Physiology and Allied Science.

[19] McLellan S., Muddimer A., \& Peres C S., (2012), The Effect of Experience on System Usability Scale Ratings. journal of Usability Studies, 7(2), P 56-67.

[20] Thomsen, C., (2012), Database programming with C\#, P (60), Apress, 2002.

[21] Rackley S A., (2011), Wireless networking technology: From principles to successful implementation, P (45), Oxford: Elsevier.

[22] The Iraqi Legislation, (2011), Directory of state employees and the public sector in the Republic of Iraq, Baghdad: The Iraqi Legislation newspaper.

[23] Shalaa, R A A A., 2011, The role of cloud accounting information systems in raising the efficiency of financial performance, P (30), The Interior Ministry case study, Khartoum: Sudan University for science and technology.

[24] Almutiry, H F D., (2010), Applying human resource accounting systems in public joint-stock companies in the state of Kuwait, justifications, principles, and obstacles, Master thesis, Kuwait: Middle East University, Business College, Accounting department.

[25] Fadhel Allah, J S., (2010), designing and preparing database management a system for Baghdad university employees, Technical magazine, 23(6), P (72-56).

[26] Aldaya, M Y., 2009, The effect of using accounting information systems on the quality of financial data in the service sector in Gaza (case study), P (33), Gaza: Islamic university, College of Commerce, Department of Accounting and Finance. 